\title{
Wayside Bearing Fault Diagnosis Based on Envelope Analysis Paved with Time-Domain Interpolation Resampling and Weighted-Correlation-Coefficient-Guided Stochastic Resonance
}

\author{
Yongbin Liu, ${ }^{1,2}$ Qiang Qian, ${ }^{1}$ Fang Liu, ${ }^{1,3}$ Siliang Lu, ${ }^{1,3}$ Qingbo He, ${ }^{2}$ and Jiwen Zhao ${ }^{1,3}$ \\ ${ }^{1}$ School of Electrical Engineering and Automation, Anhui University, Hefei, Anhui 230601, China \\ ${ }^{2}$ Department of Precision Machinery and Precision Instrumentation, University of Science and Technology of China, \\ Hefei, Anhui 230026, China \\ ${ }^{3}$ National Engineering Laboratory of Energy-Saving Motor \& Control Technology, Anhui University, Hefei, Anhui 230601, China
}

Correspondence should be addressed to Fang Liu; ufun@ahu.edu.cn and Siliang Lu; lusliang@mail.ustc.edu.cn

Received 8 July 2016; Revised 13 October 2016; Accepted 30 October 2016; Published 4 January 2017

Academic Editor: Mario Terzo

Copyright (C) 2017 Yongbin Liu et al. This is an open access article distributed under the Creative Commons Attribution License, which permits unrestricted use, distribution, and reproduction in any medium, provided the original work is properly cited.

\begin{abstract}
Envelope spectrum analysis is a simple, effective, and classic method for bearing fault identification. However, in the wayside acoustic health monitoring system, owing to the high relative moving speed between the railway vehicle and the wayside mounted microphone, the recorded signal is embedded with Doppler effect, which brings in shift and expansion of the bearing fault characteristic frequency (FCF). What is more, the background noise is relatively heavy, which makes it difficult to identify the FCF. To solve the two problems, this study introduces solutions for the wayside acoustic fault diagnosis of train bearing based on Doppler effect reduction using the improved time-domain interpolation resampling (TIR) method and diagnosis-relevant information enhancement using Weighted-Correlation-Coefficient-Guided Stochastic Resonance (WCCSR) method. First, the traditional TIR method is improved by incorporating the original method with kinematic parameter estimation based on timefrequency analysis and curve fitting. Based on the estimated parameters, the Doppler effect is removed using the TIR easily. Second, WCCSR is employed to enhance the diagnosis-relevant period signal component in the obtained Doppler-free signal. Finally, paved with the above two procedures, the local fault is identified using envelope spectrum analysis. Simulated and experimental cases have verified the effectiveness of the proposed method.
\end{abstract}

\section{Introduction}

Wheel bearings are one of the key elements of railway vehicles and their failure is one of the most frequent reasons for railway transport accident. It is of great importance to ensure the health condition of them [1]. Wayside acoustic monitoring technique is one of the promising online health monitoring techniques for wheel bearings because of its noncontact measurement and the ability of early fault detection as well as low cost [2-4]. This technique is based on the assumption that diagnosis-relevant information is stored in the acoustic signals of bearings. With signal processing techniques, fault characteristic information can be extracted from the wayside recorded acoustic bearing signals [5].

When a local defect appears inside the roller bearing, repeated spikes in time waveform excite the structure's resonance to high frequency band. By means of amplitude demodulation (like Hilbert transform) and the Fourier transform, the bearing fault characteristic frequency (FCF) on a demodulated spectrum can be identified [6,7]. That is the basic idea of envelope spectrum analysis. Due to the advantage of universality, it has gained a leading role among various methods for bearing diagnosis in the last years. However, due to the high relative speed between the railway vehicle and the microphone, the wayside recorded signal is embedded with Doppler effect, which causes high frequency shift in the resonance frequency band. As a result, the phenomenon of FCF shift and expansion appears in the envelope spectrum reducing the diagnosis accuracy and effectiveness of the envelope spectrum analysis.

Generally, there are two methods to remove the Doppler effect embedded in wayside acoustic signals; one is the 
dynamic signal resampling method, and the other is the time-domain interpolation resampling (TIR) method [8]. The basic idea of the first method is to resample the Dopplershifted signal with a time-varying sampling frequency set according to the local instantaneous frequency (IF). This method can be implemented based on the signal itself and there are many ways to achieve IF estimation (IFE). However, the effectiveness and accuracy highly depend on the IF estimation accuracy. And the IFE procedure usually needs artificial process. For example, in Zhang's method [9], a small range of frequencies have to be selected artificially around the peak point of the frequency before time-frequency ridge line searching. In his method, the starting point for ridge line searching is chosen as the center point of the frequency band at the beginning of the variation curve which is determined by visual inspection. And the frequency area for ridge line searching is also selected manually. What is more, in practice, there are many other sound sources in the moving railway vehicles and the background noise is heavy which will affect the effectiveness of these methods. On the other hand, the amplitude modulation problem is also not considered in these methods. The basic idea of the second method is to restore the amplitude of the source signal using interpolation methods. It is absolutely a time-domain method; thus, the accuracy of the Doppler effect elimination can be improved. And both time- and frequency-domain structures can be corrected. However, the time series for interpolation is calculated based on the kinematic parameters that should be known beforehand or measured by sensors. This method cannot be implemented based on the signal itself. A parameter estimation method is proposed by Liu based on Doppler-let transform. The parameters can be estimated through correlation analysis between the wayside signal and a parameterized atom. The amount of computation is heavy and the effectiveness will be affected by the background noise and the other sound sources of the railway vehicles. In this study, a new technique using TF ridge extraction and curve fitting method is applied to estimate the parameters to improve the TIR method. Kinematic parameters are estimated prior to the interpolation process. In the proposed method, before TF ridge extraction, a strategy of background noise elimination and a strategy of interference elements elimination are provided to highlight the interested TF ridge line. The proposed two strategies can improve the accuracy of the TF ridge extraction and the program automation of arithmetic.

Another challenge is to enhance the weak diagnosisrelevant information from heavy background noise. There are many other sound sources in vehicles beside the wheel bearings, such as rolling noise, mechanical equipment noise, aerodynamic noise, and structural borne noise. Although the Doppler effect has been removed, identifying the FCF from the Doppler-free signal still remains difficult. In the last years, many methods for enhancing the diagnosis-relevant information of rotating machinery, such as digital filters $[10,11]$, wavelet transform [12-14], stochastic resonance (SR) [15, 16], manifold learning $[17,18]$, and morphological analysis [19, 20], have been invented. Among them, SR can utilize noise to enhance the weak periodic signal, which benefits weak signal

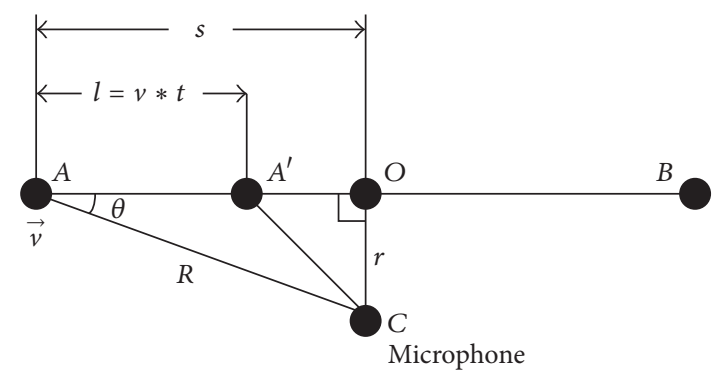

FIGURE 1: Basic kinematic model of the wayside acoustic bearing monitoring system.

detection especially when the signal bandwidth is overlapped with the noise bandwidth. Utilizing this advantage, SRbased signal enhancement methods have been widely used in bearing fault diagnosis. In the SR method, a criterion is required to guide parameters tuning to obtain the optimal output. Weighted correlation coefficient (WCC) is a criterion first introduced by Wang et al. [21]. It is a product of the power spectrum kurtosis (PSK) of the output signal and the correlation coefficient (CC) between the input and the output signals. Comparing with the traditional signal-tonoise ratio (SNR) criterion, the WCC is a criterion in both time and frequency domain. This study proposes a diagnosis information enhancement process based on WCC-guided stochastic resonance (WCCSR) after the Doppler effect is removed. The periodicity of the weak Doppler-free signal can be improved so that the FCF can be identified more clearly.

The proposed solution utilizes the Doppler effect reduction and information enhancement for noise treatment. The improvement of the TIR method allows fault diagnosis even without preknowledge of kinematic parameters. And the WCCSR can further enhance the FCF identification. Simulated and experimental case studies are conducted to verify the effectiveness of the introduced methods.

The remainder of this paper is organized as follows. The basic kinematic model of the wayside acoustic bearing monitoring system and the Doppler effect reduction using the traditional TIR are introduced in Section 2. Section 3 provides the kinematic parameter estimation based on TF ridge extraction. A simulated verification is also provided in this section. Section 4 is introduction of the diagnosis-relevant information enhancement using the WCCSR method. Section 5 discusses an experimental verification test using defective locomotive roller bearings with outer- and inner-race defects. Finally, Section 6 concludes this study.

\section{Doppler Effect Reduction Using Traditional TIR}

2.1. Kinematic Model. According to the actual situation, the kinematic model of the wayside acoustic health monitoring can be created as shown in Figure 1, where $v$ stands for the velocity of the source point. The Mach number is $M=v / c$ that is lower than 1 in practice. $c$ denotes the wave velocity in air. $R$ denotes the distance between the bearing sound source and the microphone during the movement, and $R$ 


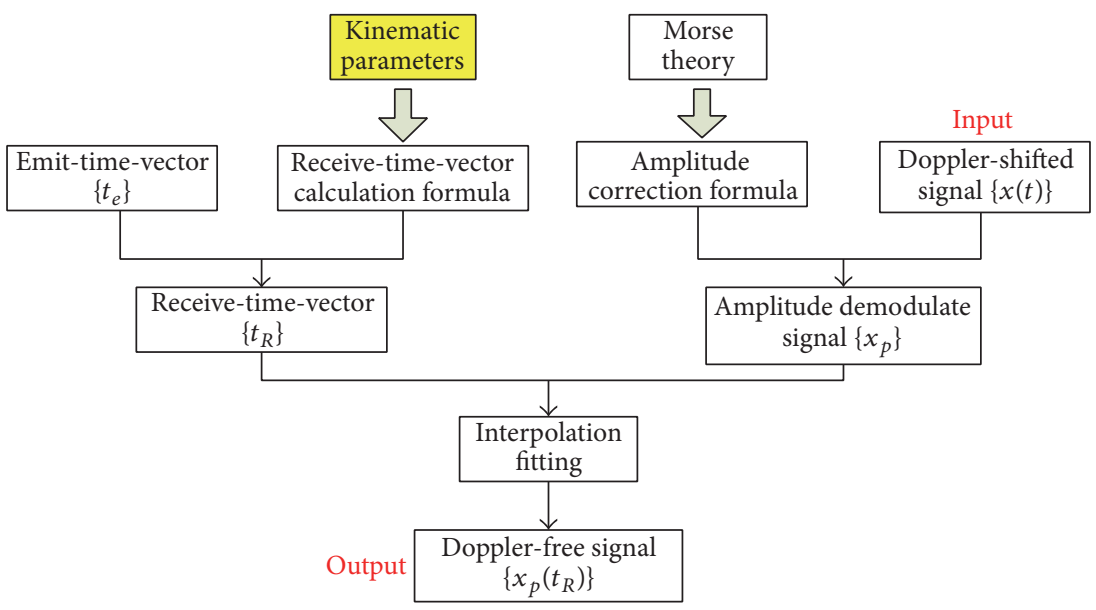

FIgURE 2: Framework of the traditional TIR.

is a time-varying variable. $r$ is the distance between the microphone and moving path of the sound source. $S$ stands for the horizontal distance between the sound source and the microphone at the initial time. $f_{0}$ is defined as the center frequency of distortion signal. The kinematic model can be described by the following parameters: $\{v, r, s\}$.

Assume the sound source emits a pure harmonic signal with center frequency of $f_{0}$. The frequency of the received signal can be described as follows which is derived based on Morse acoustic theory and kinematic relations [22]:

$$
f(t)=f_{0} \cdot \frac{M(s-v t)+\sqrt{(s-v t)^{2}+\left(1-M^{2}\right) r^{2}}}{\left(1-M^{2}\right) \sqrt{(s-v t)^{2}+\left(1-M^{2}\right) r^{2}}} .
$$

2.2. TIR. The framework of the TIR is depicted in Figure 2; the steps are described as follows [8].

(1) Receiving-Time Vector Calculation. The emitting-time vector is $\left\{t_{e}\right\}=\left\{0,1 / f_{s}, 2 / f_{s}, \ldots,(N-1) / f_{s}\right\}$, where $f_{s}$ stands for the resampling frequency and $N$ represents the length of the Doppler-shifted signal. The receiving-time vector $\left\{t_{R}\right\}$ is calculated using the receive-time-vector calculation formula as in (2) which is derived by kinematic model analyses. $r$ stands for the distance between the microphone and the middle of the track, and $c$ denotes the speed of sound propagation in air (established as $c=340 \mathrm{~m} / \mathrm{s}$ ).

$$
t_{R}=t+\frac{\sqrt{r^{2}+(s-v t)^{2}}}{c} .
$$

(2) Amplitude Demodulation. The amplitude of the Dopplershifted signal $\left\{x_{t}\right\}$ is restored according to the amplitude demodulation function described as follows:

$$
A_{\mathrm{dem}}=A^{-1}=\frac{R(1-M \cos \theta)^{2}}{r} \text {. }
$$

(3) Signal Restoration Using Interpolation. The amplitude demodulated signal is interpolated using the receiving-time vector $\left\{t_{R}\right\}$ calculated in Step (1). The restored signal can be expressed as $\left\{x_{p}\left(t_{R}\right)\right\}$.

In this method, the receiving-time vector is calculated based on the kinematic parameters $\{v, r, s\}$ that should be known beforehand. To overcome this limitation, kinematic parameter estimation based on the signal itself prior to the interpolation process is introduced in the next section to further improve the TIR method.

\section{Kinematic Parameter Estimation}

3.1. Scheme. Doppler effect phenomenon is a nonlinear timevarying nature. The time-varying characteristic depends on the kinematic parameters. The internal connection between the time-varying characteristic and the parameters can be described by (1) which is derived based on the Morse acoustic theory. So the kinematic parameters can be estimated through curve fitting on the TF ridge line of the recorded bearing signal.

Based on this idea, detailed steps of the estimation procedure are shown in Figure 3. First, a band-pass filter is applied on the recorded bearing signal. Then, the time-frequency distribution (TFD) should be calculated. There are several ways to obtain good results of TFD [3], such as wavelet transform [23, 24], short-time Fourier transform (STFT) [25, 26], Wigner-Ville distribution [27, 28], and Hilbert-Huang transform [29, 30]. Among them, the STFT is sophisticated and effective in many situations, so it is employed in this study to obtain the TFD of the Doppler-shifted bearing signal. Considering that there are many other sound sources and the background noise is heavy, a strategy of background noise elimination and a strategy of interference elements elimination are provided to highlight the interested timefrequency ridge line. Finally, the kinematic parameters of sound source can be obtained through curve fitting using (1) on the TF ridge line based on the least-square method. After the kinematic parameters are determined, the receivingtime vector and the amplitude demodulation function are obtained according to the theory of TIR. The receivingtime vector is applied to resample the distortion signal 
(1) Signal filtering and TFD

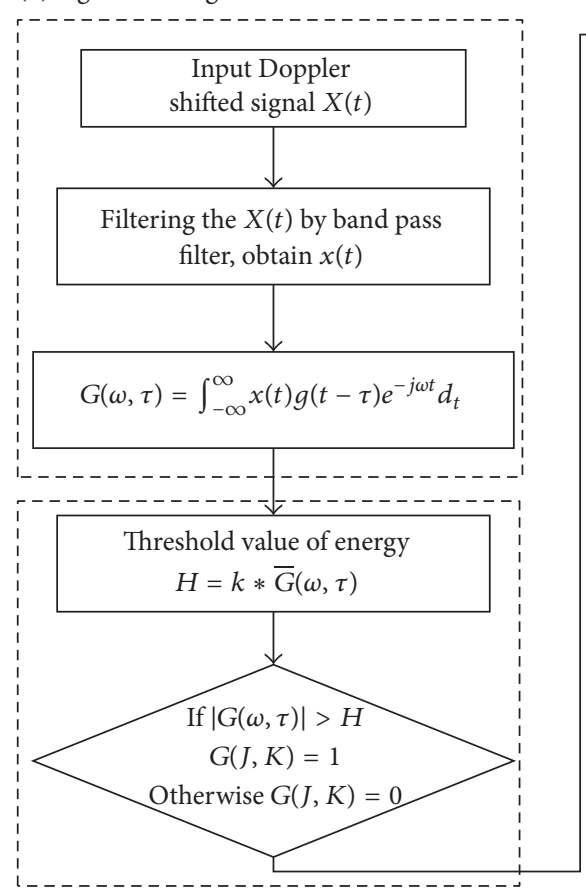

(2) Eliminate background noise
(3) Eliminate the interference element

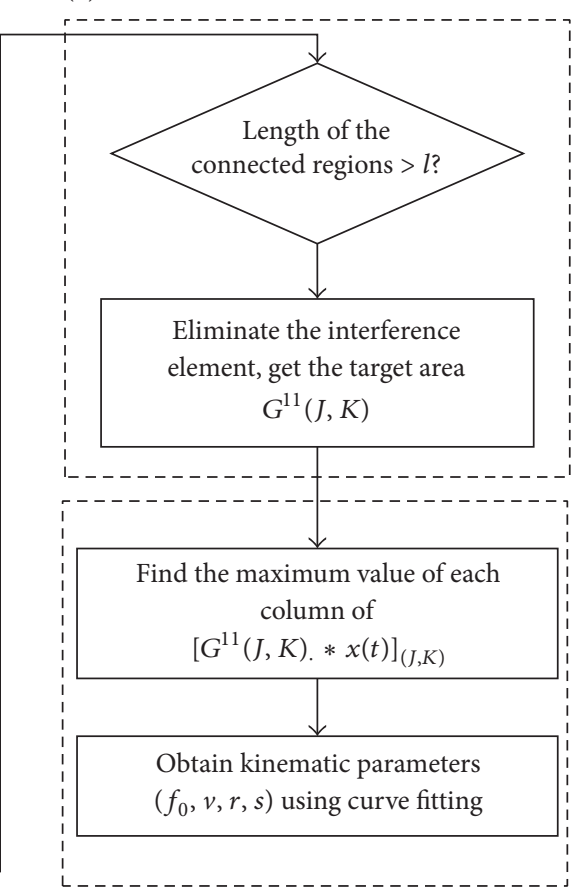

(4) Kinematics parameter identification

FIGURE 3: Flowchart of the algorithm of kinematic parameters estimation.

to eliminate the distortion in the time domain, and the amplitude demodulation function is used to correct the signal amplitude. The flowchart of kinematic parameter estimation is shown in Figure 3. The process is roughly divided into four parts, namely, band-pass filtering and TFD, elimination of background noise, elimination of interference element, and kinematic parameter identification. Assume the recorded Doppler-shifted signal is $X(t)$; the steps of the parameters estimation are as follows.

(1) Band-Pass Filtering and TFD. $x(t)$ is obtained by using Butterworth band-pass filter on $X(t)$. The TFD of $x(t)$ is obtained as follows:

$$
G(\omega, \tau)=\int_{-\infty}^{\infty} x(t) g(t-\tau) e^{-j \omega t} d_{t}
$$

(2) Elimination of Background Noise. $x(t)$ is not only polluted by noise but also interfered with by multiple frequency components. An energy threshold $H$ expressed as follows is set to distinguish between valid and noisy components:

$$
H=k * \bar{G}(\omega, \tau) \text {, }
$$

where $k$ is the weight that is determined by experience. $\bar{G}(\omega, \tau)$ is the average energy of the TF matrix. $G(J, K)$ represents the TF matrix of $G(\omega, \tau)$. This matrix has $J$ rows and $K$ columns. The noisy components can be eliminated using

$$
G(J, K)= \begin{cases}1 & \text { if }|G(\omega, \tau)| \geq H \\ 0 & \text { else. }\end{cases}
$$

After this step, all elements in this TF matrix $G(J, K)$ are labeled by 1 or 0 . Then, the connected regions are determined according to the eight-connected-region algorithm [31, 32]. The detail steps can be described as follows:

(1) If the values of $G(J, K-1), G(J-1, K-1), G(J-$ $1, K)$, and $G(J-1, K+1)$ are zero, then $G(J, K)$ is the beginning of a connected region.

(2) If the values of $G(J, K-1)$ and $G(J-1, K+1)$ are not zero, then the value of $G(J, K)$ will be set as the minimum between the two points, and the values of $G(J, K-1)$ and $G(J-1, K+1)$ are also set as the minimum.

(3) If the values of $G(J-1, K-1)$ and $G(J-1, K+1)$ are not zero, then the value of $G(J, K)$ will be set as the minimum between the two points, and the values of $G(J-1, K-1)$ and $G(J-1, K+1)$ are also set as the minimum.

(4) Otherwise, the order of $G(J, K-1), G(J-1, K-1)$, $G(J-1, K)$, and $G(J-1, K+1)$ is used to label $G(J, K)$.

The TF matrix $G(J, K)$ can be divided into several connected regions $G^{1}(J, K)$ through the above steps. The pixels labeled as 0 are the background, the pixels labeled as 1 make 


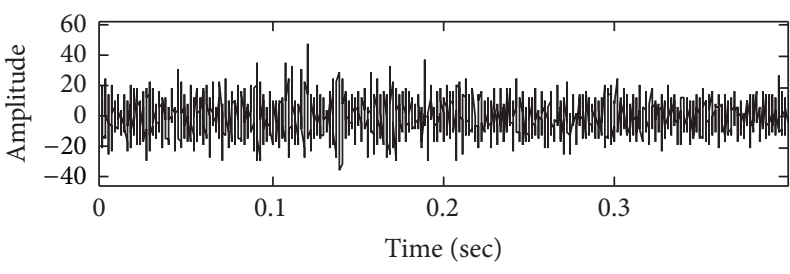

(a)

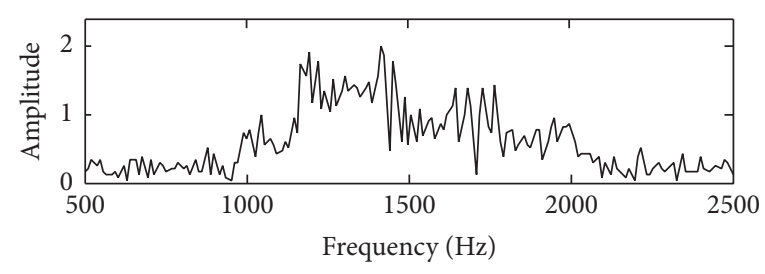

(b)

Figure 4: (a) Waveform of the simulated Doppler-shifted signal; (b) FFT spectrum.

up one object, the pixels labeled as 2 make up the second object, and so on. The value of each region will be set as the corresponding label to the next step of processing.

(3) Elimination of Interference Elements. $G^{1}(J, K)$ can be eliminated when the length of $G^{1}(J, K)$ is shorter than the length threshold $l$ which is determined by inspection, and the remaining area can be reserved as the target area $G^{11}(J, K)$.

$$
G^{11}(J, K)=\left\{\begin{array}{lc}
1 & \text { Length }\left(G^{1}(J, K)\right) \geq l \\
0 & \text { Length }\left(G^{1}(J, K)\right)<l
\end{array}\right.
$$

(4) Kinematic Parameter Identification. The local maximum is searched in each column of $\left[G^{11}(J, K) * G(\omega, \tau)\right]_{(J, K)}$. The TF ridge line can be attained by connecting all of the maximums of each column. Equation (1) is applied to curve fitting for the kinematic parameters $\left(f_{0}, v, r, s\right)$.

Through the above four steps, we can obtain the kinematic parameters based on the wayside recorded signal with Doppler effect. Therefore, their values are not required to measure in advance. Such parameters are an important premise for the TIR method. This process is effective to overcome the limitation of traditional TIR. Thus, the agility and convenience of TIR can be improved. A simulated verification is carried out in the next section.

3.2. Simulated Verification. In this case, a simulated Dopplershifted signal with four characteristic frequencies is analyzed to verify the effectiveness of the proposed parameters estimation method. The simulated signal is generated referring to the model in Figure 1 with the following assumption: $s=$ $1.5 \mathrm{~m}, r=1 \mathrm{~m}, v=40 \mathrm{~m} / \mathrm{s}$, and $c=340 \mathrm{~m} / \mathrm{s}$. The simulated Doppler-shifted signal can be expressed as [8]

$$
\begin{aligned}
x(t)= & \sum_{i=1}^{4} \frac{2 \pi q_{i} f_{i}}{4 \pi R(1-M \cos \theta)^{2}} \cos \left(2 \pi f_{i}\left(t-\frac{R}{c}\right)\right) \\
& +n(t) .
\end{aligned}
$$

The four frequency components are $f_{1}=1000 \mathrm{~Hz}, f_{2}=$ $1300 \mathrm{~Hz}, f_{3}=1500 \mathrm{~Hz}$, and $f_{4}=1700 \mathrm{~Hz}$, which are close to the characteristic frequency of the defective bearing in the subsequent experimental cases. The signal intensity is set as $q_{1}=0.7, q_{2}=1.7, q_{3}=0.9$, and $q_{4}=1.1$. The sampling frequency is set as $50 \mathrm{kHz}$ and the length of the simulated
TABLE 1: Results of parameters estimation.

\begin{tabular}{lcccc}
\hline Parameters & $f_{0}$ & $v$ & $s$ & $r$ \\
\hline Actual value & $1500 \mathrm{~Hz}$ & $40 \mathrm{~m} / \mathrm{s}$ & $1.5 \mathrm{~m}$ & $1 \mathrm{~m}$ \\
Fitted value & $1500 \mathrm{HZ}$ & $40.02 \mathrm{~m} / \mathrm{s}$ & $1.49 \mathrm{~m}$ & $1 \mathrm{~m}$ \\
Error $(\%)$ & 0 & 0.05 & 0.67 & 0 \\
\hline
\end{tabular}

signal is $N=4000$. A random noise $n(t)$ with intensity $q_{n}=5$ is added to the signal to simulate the actual situation.

The simulated signal is demonstrated in Figure 4(a), with its FFT spectrum in Figure 4(b). According to the method introduced in Section 2, the TFD of the band-pass signal is obtained using STFT and shown in Figure 5(a). Then, the background noise is eliminated using a threshold value of $H=1507.1(k=7, \bar{G}(\omega, \tau)=215.3)$. The pixels of the TFD matrix are labeled and classified to different regions using eight-connected-region algorithm. The result is shown in Figure 5(b). The interference elements are then eliminated according to the length of the region. The minimum length $l$ is set to be 50 here. The TFD is updated in Figure 5(c). It can be seen that the single main TF curve of the Doppler-shifted signal has be obtained. Finally, as shown in Figure 5(d), the TF ridge line is attained by connecting all of the maximums of each column of the TF matrix. The kinematic parameters can then be estimated by using curve fitting based on the least-square method. The effect of the curve fitting and the parameters obtained from the curve fitting are shown in Figure 6 and Table 1, respectively.

Paved with the estimated parameters, the Doppler-shifted signal is processed by TIR. The amplitude demodulation function is calculated according to (3) and is demonstrated in Figure 7(a). This function is used to correct the signal amplitude. On the time dimension, the receiving-time vector is calculated using (2), which is demonstrated in Figure 7(b). The time series is used to interpolate the distortion signal to eliminate the distortion in the time domain. The TFD of the Doppler-free signal is shown in Figure 7(c). It can be seen that TF curves are flat which indicates that the Doppler effect is totally removed.

The simulation in this section has verified the effectiveness of the proposed parameters estimation method based on TF ridge line extraction and curve fitting as well as the Doppler effect reduction using TIR. After this process, however, in practice, the FCF is still difficult to be identified. Hence, the information enhancement method using WCCSR is introduced in the next part. 


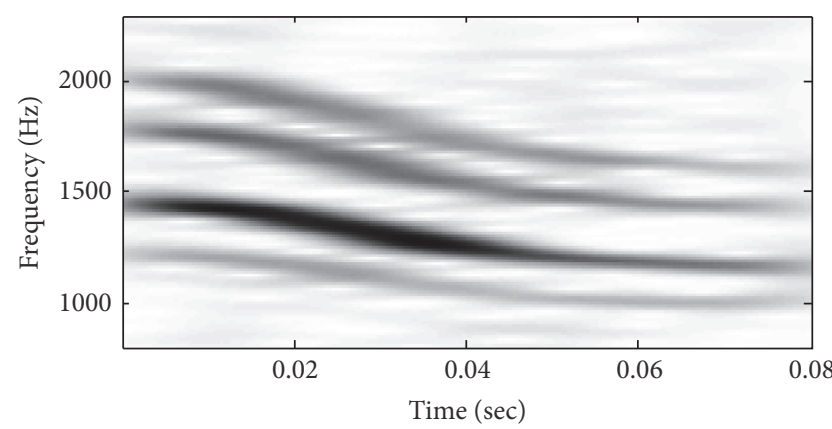

(a)

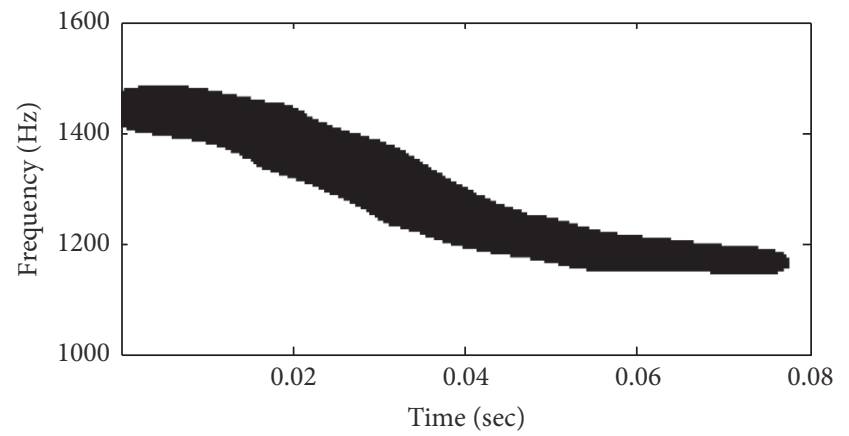

(c)

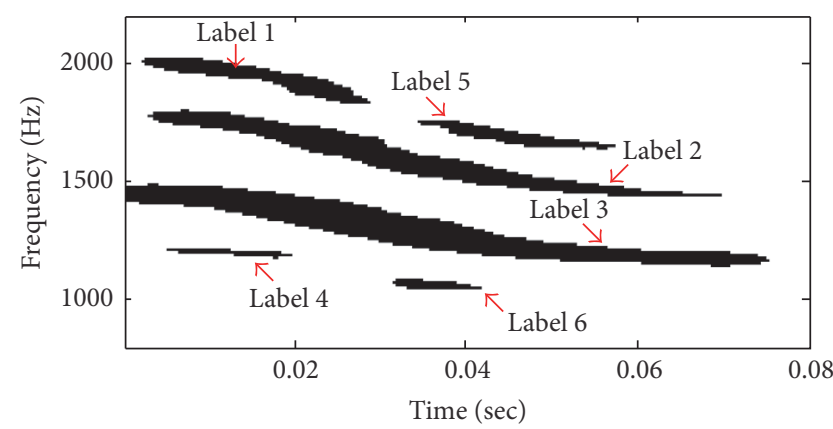

(b)

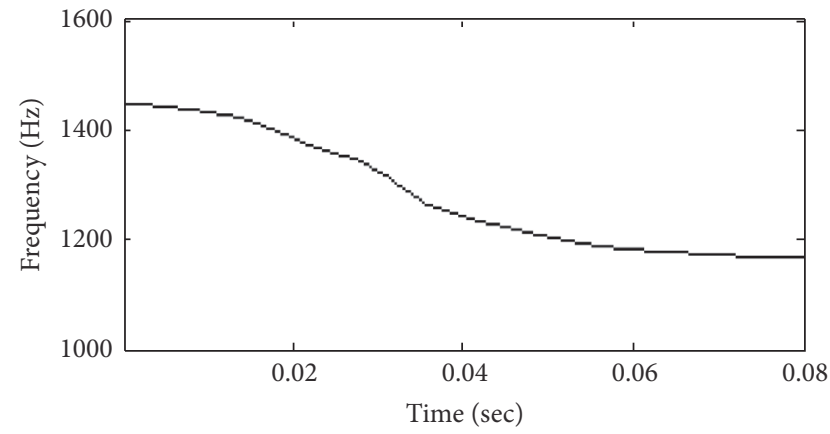

(d)

Figure 5: Process of ridge line extraction: (a) band-pass signal; (b) background noise cancellation; (c) interference element cancellation; (d) TF ridge line extraction.

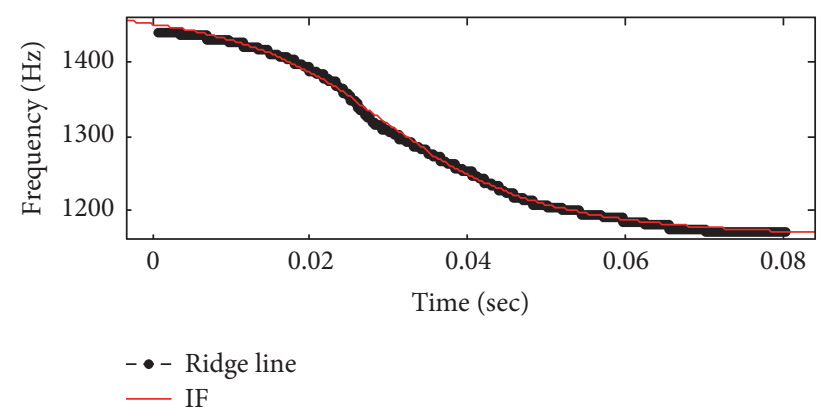

FIGURE 6: Parameter estimation using curve fitting.

\section{Information Enhancement Using WCCSR}

After the Doppler effect is eliminated, however, there are many other sound sources on the railway vehicle and the background noise is heavy. The diagnosis-relevant information is submerged, so it is difficult to identify the FCF directly. SR has been widely applied for identifying the FCF in mechanical system. This method can enhance the output signal of a nonlinear dynamic system by means of noise addition to the system. Traditional SR methods used SNR as the criterion to tune the parameters $[33,34]$. When the driving frequency is unknown, the SR methods cannot work favorably. The WCCSR utilizes WCC as criterion to guide parameters tuning. The WCC criterion concerns the signal characteristics in both time and frequency domains and it has been proved effective in bearing fault diagnosis [21]. In this paper, the WCCSR is applied to further analyze the
Doppler-free signal to enhance the weak diagnosis-relevant information.

4.1. WCCSR Method. The SR model can be described by

$$
\frac{d^{2} x}{d t^{2}}=a x-b x^{3}-\gamma \frac{d x}{d t}+S(t)+N(t)
$$

where $N(t)=\sqrt{2 D} \xi(t)$ satisfies $\langle N(t) N(t+\tau)\rangle=2 D \delta(t), D$ is noise intensity, $\xi(t)$ is additive Gaussian white noise (AGWN) with zero mean and unit variation, $S(t)=A \cos \left(2 \pi f_{d} t+\varphi\right)$ is the periodic signal with $A, f_{d}$, and $\varphi$ being the amplitude, the driving frequency, and the phase, respectively, $\gamma$ is the damping function, and $a$ and $b$ are the bistable potential parameters with positive real values. Subsequently, the system of SNR can be deduced as

SNR

$$
\begin{aligned}
= & \frac{a A^{2} \sqrt{2 a}}{4 b D^{2}} \exp \left(-\frac{a^{2}}{4 b D}\right) \\
& \cdot\left[1-\frac{2 a^{2} A^{2} \exp \left(-a^{2} / 2 b D\right)}{4 a b D^{2} \exp \left(-a^{2} / 2 b D\right)+2 b D^{2} \pi^{2} \Omega^{2}}\right]^{-1} .
\end{aligned}
$$

The SNR of the output signal is a nonlinear function dominated by both the input signal and the system parameters. The optimal output signal can be achieved by tuning the system parameters for the deterministic input signal, so that weak periodic signal enhancement can be 


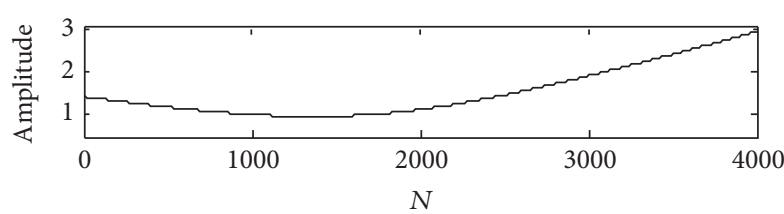

(a)

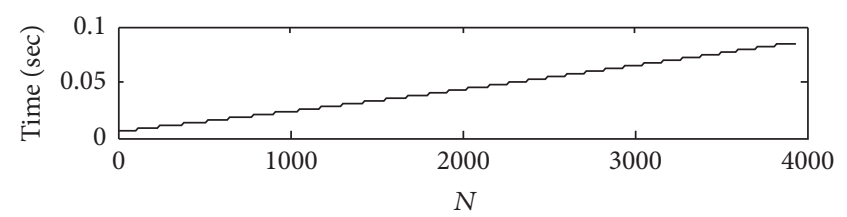

(b)

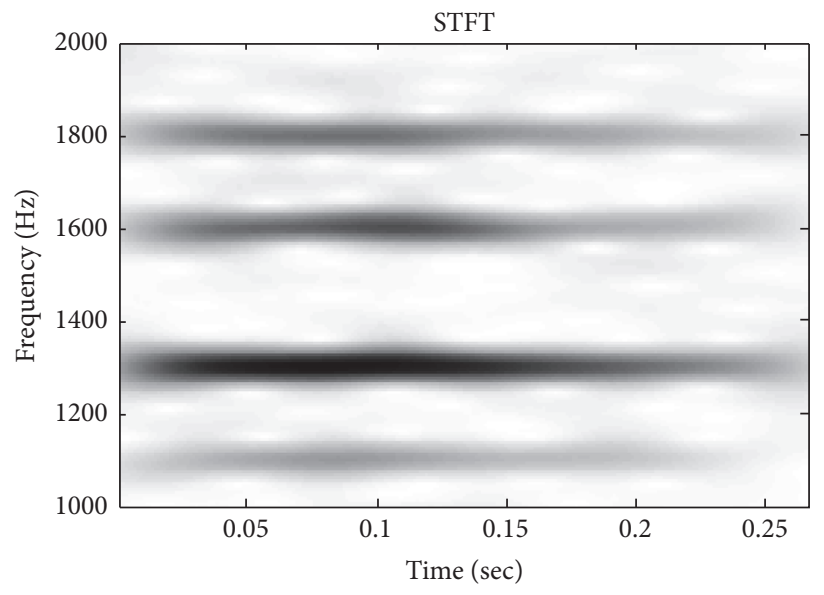

(c)

FIgURE 7: (a) Amplitude demodulation function; (b) receiving-time vector; (c) STFT of the Doppler-free signal.

accomplished using SR. In practice, (9) can be solved by using the classical fourth-order Runge-Kutta equation as shown in the following:

$$
\begin{aligned}
& x_{1}=-U^{\prime}(x[n])-\gamma y_{1}+S[n]+N[n] ; \\
& y_{1}=y[n] ; \\
& x_{2}=-U^{\prime}\left(x[n]+\frac{y_{1} h}{2}\right)-\gamma y_{2}+S[n]+N[n] ; \\
& y_{2}=y[n]+\frac{x_{1} h}{2} ; \\
& x_{3}=-U^{\prime}\left(x[n]+\frac{y_{2} h}{2}\right)-\gamma y_{3}+S[n+1] \\
&+N[n+1] ; y_{3}=y[n]+\frac{x_{2} h}{2} ; \\
& x_{4}=-U^{\prime}\left(x[n]+y_{3} h\right)-\gamma y_{4}+S[n+1] \\
&+N[n+1] ; \quad \\
& x[n+1]= x[n]+\frac{\left(y_{1}+2 y_{2}+2 y_{3}+y_{4}\right) h}{6} ; \\
& y[n+1]= y[n]+\frac{\left(x_{1}+2 x_{2}+2 x_{3}+x_{4}\right) h}{6} ;
\end{aligned}
$$

where $h$ is the calculation step, $x[n], y[n], S[n]$, and $N[n]$ are the discrete forms of $x(t), y(t), S(t)$, and $N(t), x(t)$ is the output signal, and $y(t)$ is the first-order derivative of $x(t)$, respectively. It can be seen from (11) that the output $x[n]$ is dominated by the parameters $(a, b, h, \gamma)$. The optimal SR output can be obtained through tuning these parameters. The WCCSR method used WCC as the criterion to tune the parameters. The WCC criterion can be described as follows:

$$
\mathrm{WCC}=\mathrm{PSK} \cdot \mathrm{CC},
$$

where PSK represents the power spectrum kurtosis of the SR output signal and CC is the correlation coefficient between the input and the SR output signals. PSK measures the kurtosis in the frequency domain while CC measures the similarity of the input signal and the output signal in time domain. A higher PSK indicates that a more pure singlefrequency signal is obtained. The CC criterion can prevent the extracted frequency component from deviating from that of the driving signal. The two criterions can be calculated via

$$
\begin{gathered}
\mathrm{PSK}=\frac{(1 /(M / 2)) \sum_{i=1}^{M / 2}\left(p x_{i}-\bar{p} \bar{x}\right)^{4}}{\left((1 /(M / 2)) \sum_{i=1}^{M / 2}\left(p x_{i}-\bar{p} \bar{x}\right)^{2}\right)^{2}} \\
\mathrm{CC}=\frac{\sum_{i=1}^{N}\left(x_{i}-\bar{x}\right)\left(z_{i}-\bar{z}\right)}{\sqrt{\sum_{i=1}^{N}\left(x_{i}-\bar{x}\right)^{2} \sum_{i=1}^{N}\left(z_{i}-\bar{z}\right)^{2}}},
\end{gathered}
$$

where $x(t)$ and $z(t)$ represent the SR output signal and input signal, respectively, $p x(f)$ denotes the power spectrum of $x(t)$, and $M$ is the length of discrete Fourier transform (DFT). $\bar{p} \bar{x}, \bar{x}$, and $\bar{z}$ represent the mean values of $p x(f), x(t)$, and $z(t)$, respectively. The objective function for tuning of parameter can be expressed as

$$
\left(h_{\mathrm{opt}}, \gamma_{\mathrm{opt}}\right)=\underset{h \in(0,+\infty), \gamma \in(0,1)}{\arg \max }(\mathrm{WCC}(h, \gamma)) .
$$




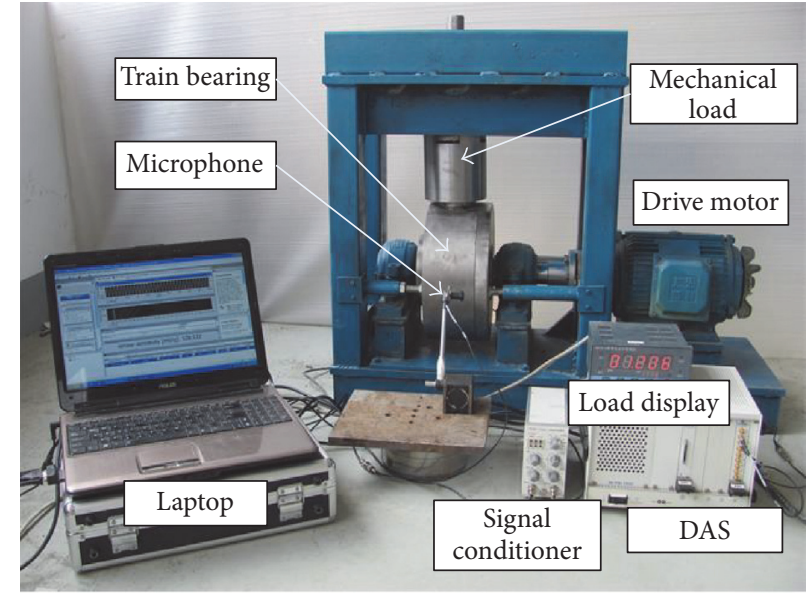

(a)

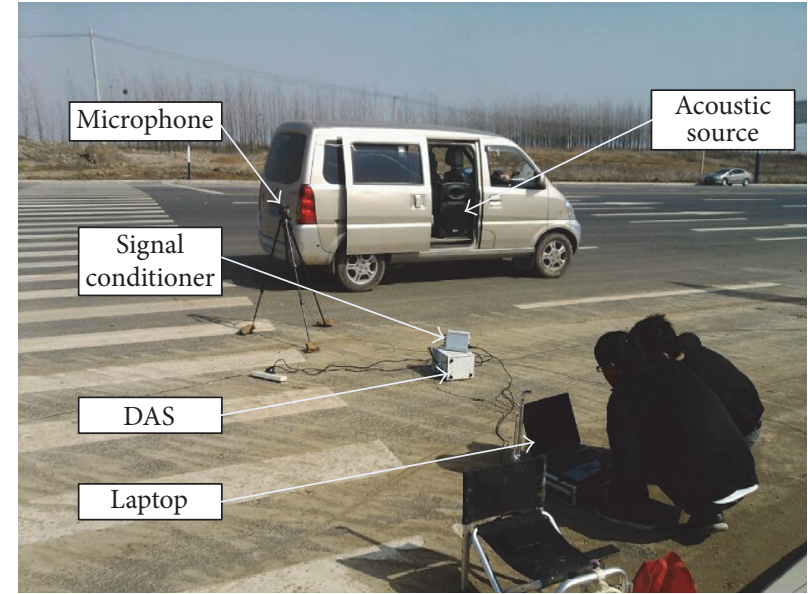

(b)

FIGURE 8: Experiment benches.

If $\gamma_{\mathrm{opt}}$ and $h_{\mathrm{opt}}$ are both within the search ranges, these two variables are the optimal parameters; otherwise, extend the range of $\gamma$ and (or) $h$; then, continue to search these optimal values.

4.2. Algorithm Flowchart. The detailed procedure of the proposed information enhancement using WCCSR for the wayside Doppler-free bearing fault signal can be summarized as follows:

(1) Demodulate the bearing signal to uncover the periodicity.

(2) Initialize the search ranges and the intervals of the SR parameters $\gamma$ and $h$.

(3) Calculate the SR outputs and the corresponding WCCs through (4) and (7), respectively, under different parameter configurations. Ascertain the optimal parameters through (8). If both $\gamma_{\mathrm{opt}}$ and $h_{\mathrm{opt}}$ are within the preset search ranges, go to Step (4); otherwise, extend the range of $\gamma$ and (or) $h$, and go back to Step (2).

(4) Construct the optimal filter using the optimal parameters $\gamma_{\text {opt }}$ and $h_{\text {opt }}$, and then obtain the optimal filtered signal.

Paved with the Doppler effect reduction process introduced in Section 3 and the diagnosis information enhancement provided in this section, the traditional envelope spectrum analysis can be applied easily to identify the FCF. Experimental signals using real defective train wheel bearings are analyzed in the next section.

\section{Experimental Case Study}

5.1. Experiment Setup and Data Acquisition. As shown in Figure 8 , two separate experiments are carried out to obtain the wayside defective bearing signal. For the details of the experiments, one can refer to [8].
In the first experiment, the acoustic signal of the tested bearing (Type: NJ(P)3226X1) was acquired by the test bench in Figure 8(a). The test bench consisted of a drive motor, two supporting pillow blocks, a tested bearing loaded on the outer race, and a mechanical radial loader. A microphone was mounted adjacent to the outer race of the tested bearing for acoustic signal measurement. The advanced data acquisition system (DAS) provided by National Instruments was used to collect data. The sampling frequency was taken as $50 \mathrm{kHz}$. The outer-race-fault frequency and the inner-race-fault frequency are $137 \mathrm{~Hz}$ and $195 \mathrm{~Hz}$, respectively.

In the second experiment, the acoustic signals acquired in the first experiment were used to acquire the signals with Doppler effect. The second experiment is illustrated in Figure 1, with the following parameters: $s=4 \mathrm{~m}, r=2 \mathrm{~m}$, and $v=30 \mathrm{~m} / \mathrm{s}$. As shown in Figure 8(b), the experiment consisted of a moving vehicle (mounted with an acoustic source), as well as the same microphone and DAS as those in the first experiment. In this experiment, the sampling frequency was also set to be $50 \mathrm{kHz}$, the velocity of the moving car was read directly from the panel of the vehicle, and the speed of sound propagation in air was set to be $c=340 \mathrm{~m} / \mathrm{s}$ conventionally.

A signal artificial crack with $0.18 \mathrm{~mm}$ width was set by a wire-electrode cutting machine on the outer and inner races, as shown in Figure 9. The Doppler-shifted bearing signals were then acquired by the aforementioned experimental steps. In the subsequent sections, the Doppler-shifted signals with the outer-race defect (size: $0.18 \mathrm{~mm}$ ) and inner-race defect (size: $0.18 \mathrm{~mm}$ ) were analyzed.

5.2. Outer-Race-Fault Signal Analysis. The outer-race-fault signal and its TFD are shown in Figure 10(a). Several TF curves with characteristic of Doppler effect phenomenon can be seen in the TFD. The envelope of the outer-race-fault signal and its FFT spectrum are shown in Figures 13(a) and 13(b). It can be seen that there is an obvious phenomenon of frequency band expansion which makes it difficult to identify the FCF clearly. So the Doppler effect should be removed 


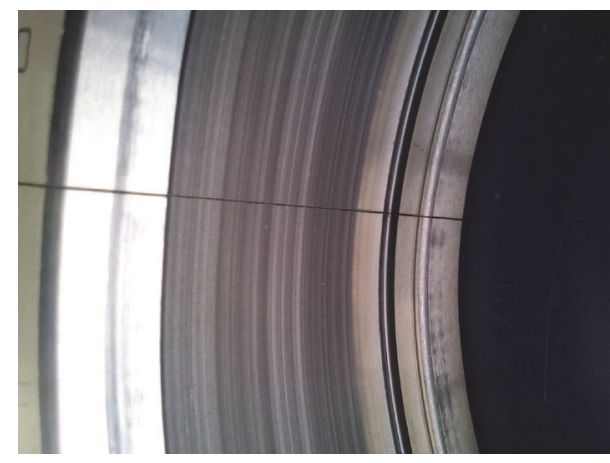

(a)

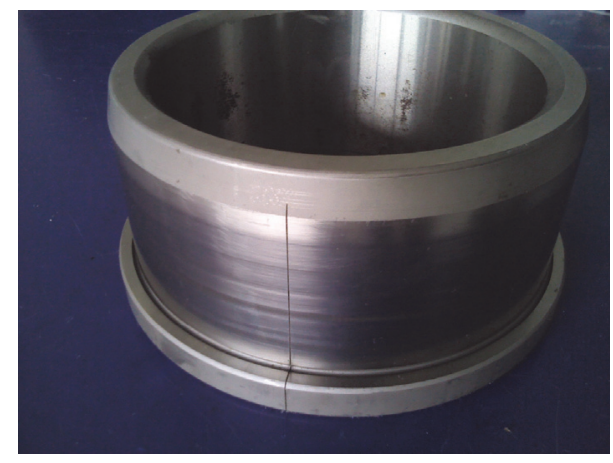

(b)

FIGURE 9: Artificial defects on the components of the bearing. (a) Defect on the outer race; (b) defect on the inner race.

firstly. The proposed parameters estimation method and the TIR method are then applied on this signal. According to the scheme introduced in Section 3, the signal is firstly filtered by a Butterworth band-pass filter with the frequency band of $[1700 \mathrm{~Hz}, 1800 \mathrm{~Hz}]$ and then handled with STFT to attain a TFD. The TFD of the band-pass signal is shown in Figure 11(a). A major TF curve can be seen in the TFD and the IF can be obtained using curve fitting of this TF curve, but there are several other TF lines which will reduce the parameters estimation accuracy of the direct curve fitting. The method explained in Section 3 is then conducted and the results are shown in Figures 11(b)-11(d). Figure 11(b) shows the background noise eliminated by setting an energy threshold of $H=0.9801(k=13, \bar{G}(\omega, \tau)=0.0075)$. The areas that satisfied the conditions were labeled. The interference elements are eliminated if the length is smaller than $l=50$. The operation process of eliminating interference element is indicated in Figures 11(b) and 11(c). The IF can be obtained by searching the local maximum in each column of the TF matrix, as shown in Figure 11(d). The parameters could be attained by curve fitting on the IF using (1).

The TF ridge line extraction by the method proposed in this study was compared with the TF ridge line extraction based on the STFT directly. The effect of the curve fitting through the first mentioned method described as method (a) is shown in Figure 12(a), and the effect of the curve fitting through the latter mentioned method described as method (b) is shown in Figure 12(b). It can be seen that the TF ridge line extracted using STFT directly fluctuates strongly in the end of the curve. The results of the estimated parameters obtained through the two methods are shown in Table 2. Results show that the proposed method achieved a higher accuracy, because the background noise and the interface elements have been eliminated through the proposed method.

The estimated parameters are then applied to TIR to reduce the Doppler effect. The amplitude demodulation function and receiving-time vector are shown in Figures 12(c) and 12(d). The waveform of the obtained Doppler-free signal and its TFD are shown in Figure 10(b). The envelope signal of the obtained Doppler-free signal and its envelope spectrum are shown in Figures 13(e) and 13(f). Comparing with the envelope spectrum of the original signal shown in Figure 13(b), the FCF of the Doppler-free signal is sharp,
TABLE 2: Results of parameters estimation by the two methods.

\begin{tabular}{lcccc}
\hline Parameters & $f_{0}$ & $v$ & $s$ & $r$ \\
\hline Actual value & $1800 \mathrm{~Hz}$ & $30 \mathrm{~m} / \mathrm{s}$ & $4 \mathrm{~m}$ & $2 \mathrm{~m}$ \\
Method (a) & $1793 \mathrm{HZ}$ & $30.01 \mathrm{~m} / \mathrm{s}$ & $3.98 \mathrm{~m}$ & $2.01 \mathrm{~m}$ \\
Error (\%) & 0.61 & 0.03 & 0.5 & 0.5 \\
Method (b) & 1786 & 29.90 & 3.91 & 2.07 \\
Error (\%) & 0.78 & 0.33 & 2.25 & 3.50 \\
\hline
\end{tabular}

narrow, and accurate which indicates that the phenomenon of FCF expansion has been eliminated. As a comparison, the parameters estimated by the traditional STFT method are also used to reduce the Doppler effect. The envelope signal of the obtained Doppler-free signal and its envelope spectrum are shown in Figures 13(c) and 13(d). It can be seen that $4 x$ and $5 \mathrm{x}$ FCF are wider than those of the proposed method which indicates that the Doppler effect has not been eliminated effectively.

After the Doppler effect reduction, however, the noise interference can also be clearly seen and there are many other frequency components which makes the identification of the FCF difficult. The calculated SNR is $-10.66 \mathrm{~dB}$. The WCCSR is then employed to amplify the FCF. The optimal output is obtained under the parameters of $h_{\mathrm{opt}}=0.027$ and $\gamma_{\mathrm{opt}}=0.017$. The output waveform and its FFT spectrum are shown in Figures 13(e) and 13(f), respectively. It can be seen that $f_{\mathrm{BPFO}}$ is highlighted and can be clearly identified after the WCCSR-based information enhancement. The SNR has been improved to be $-0.45 \mathrm{~dB}$. The results demonstrate that the diagnosis-relevant information is enhanced and the effectiveness and the diagnosis accuracy of the envelope spectrum analysis are thus improved.

5.3. Inner-Race Fault Signal Analysis. The inner-race fault signal is analyzed in this section to further verify the effectiveness of the proposed method. The waveform and the TFD are shown in Figure 14(a). There is also a Doppler effect phenomenon in the TFD, but the shape characteristic is different from that of the outer-race-fault signal. The envelope waveform and its FFT spectrum are shown in Figures 17(a) and 17(b). There is also an obvious phenomenon of frequency band expansion and the FCF is blurred. 


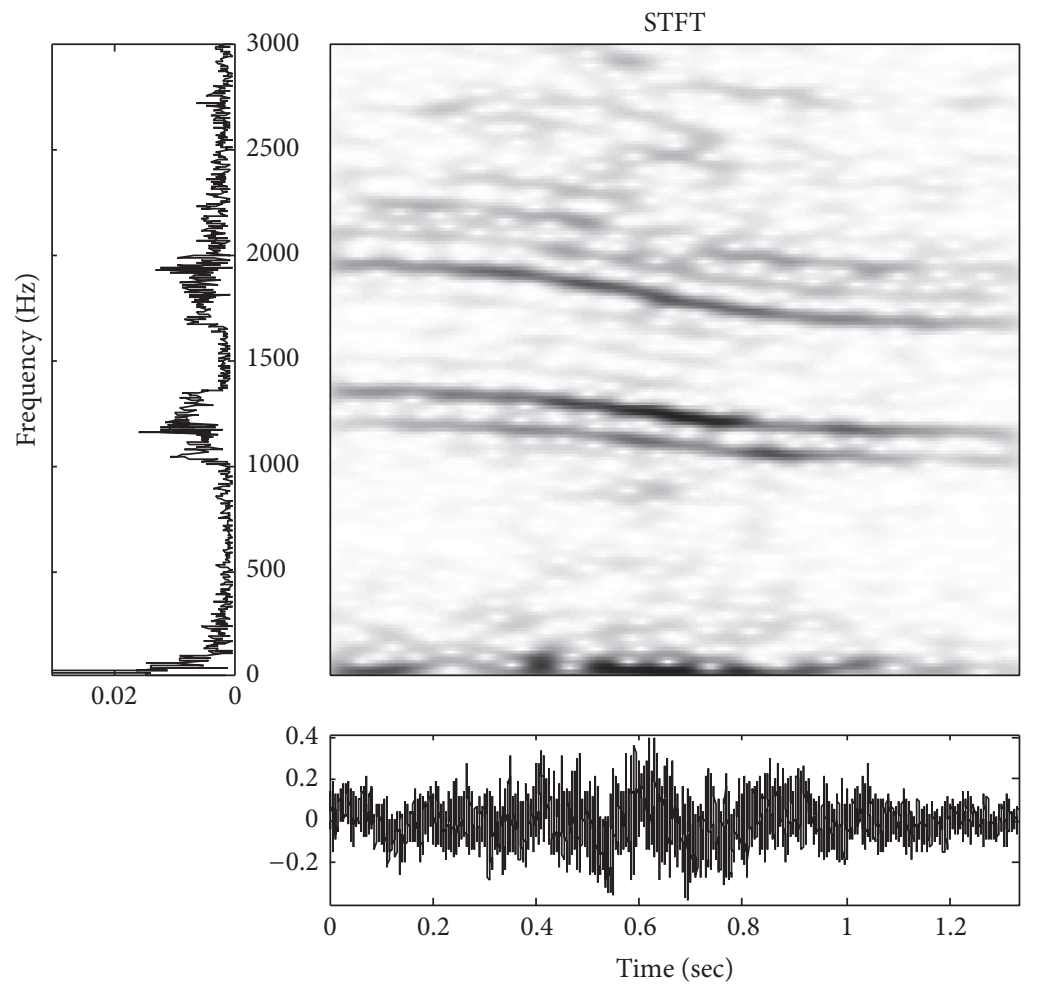

(a)
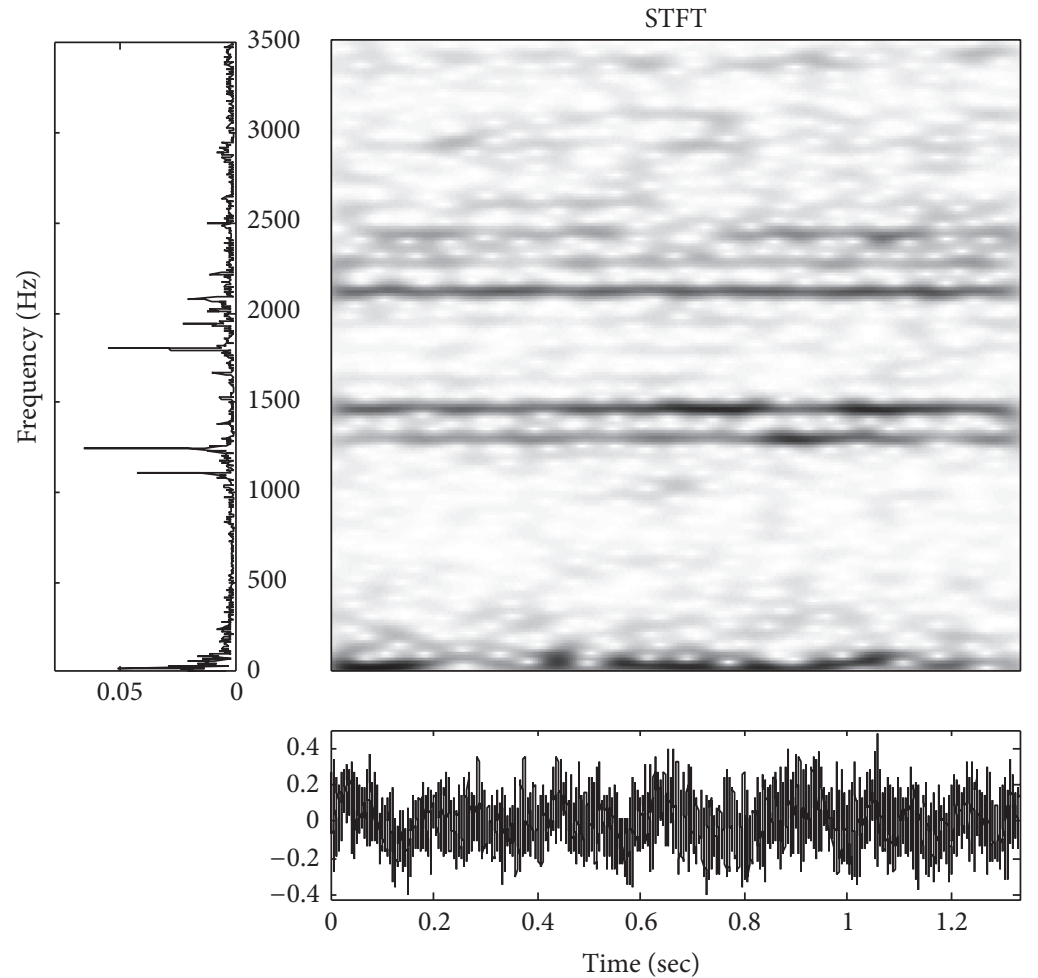

(b)

FIGURE 10: (a) Original signal; (b) Doppler-free signal. 


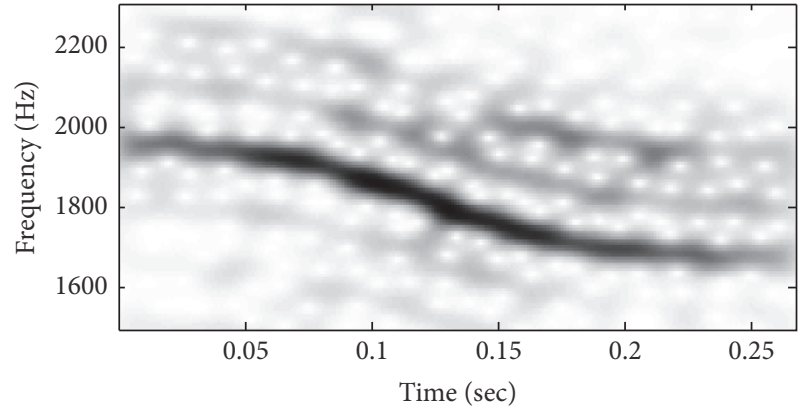

(a)

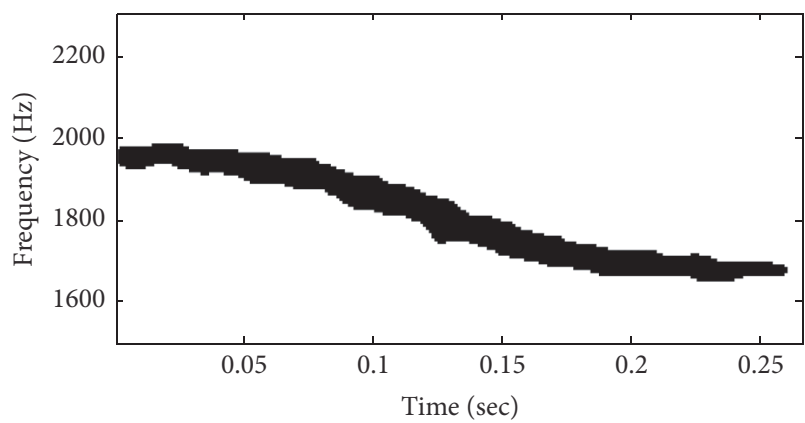

(c)

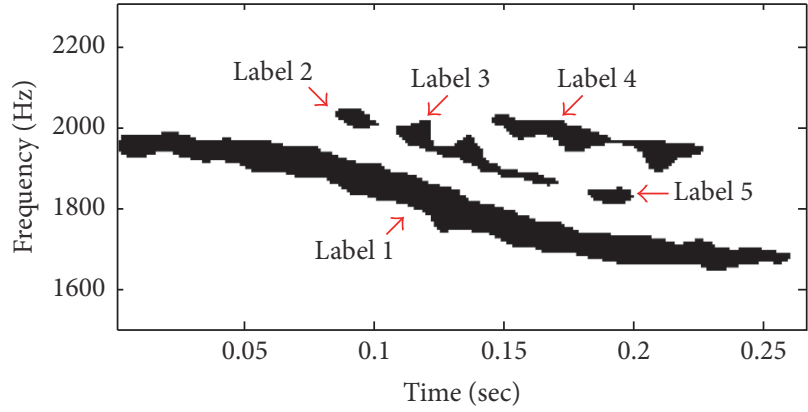

(b)

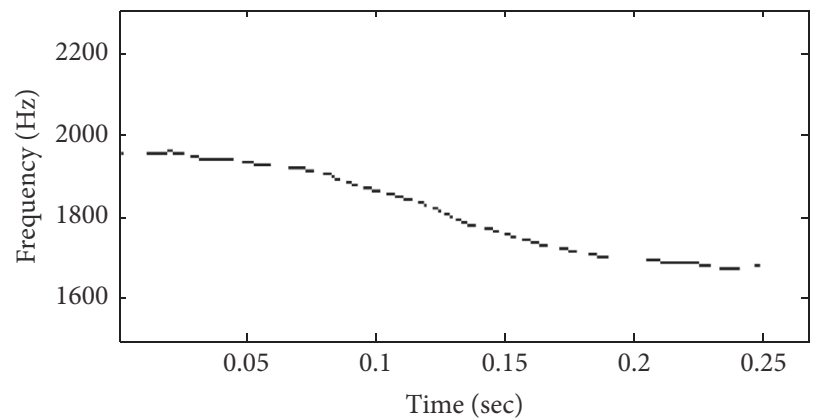

(d)

FIgURE 11: Process of ridge line extraction: (a) band-pass signal; (b) background noise cancellation; (c) interference element cancellation; (d) TF ridge line extraction.

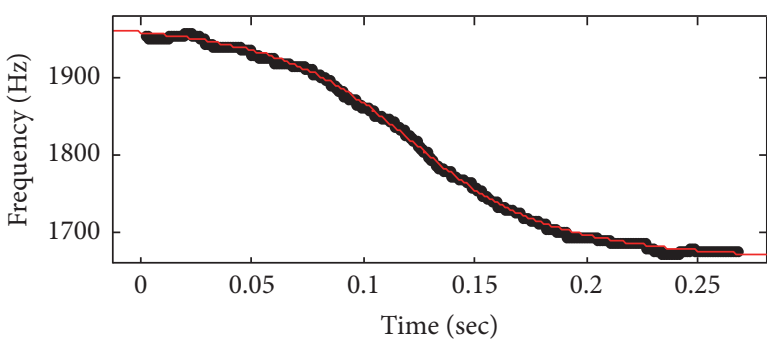

- - Ridge line

- IF

(a)

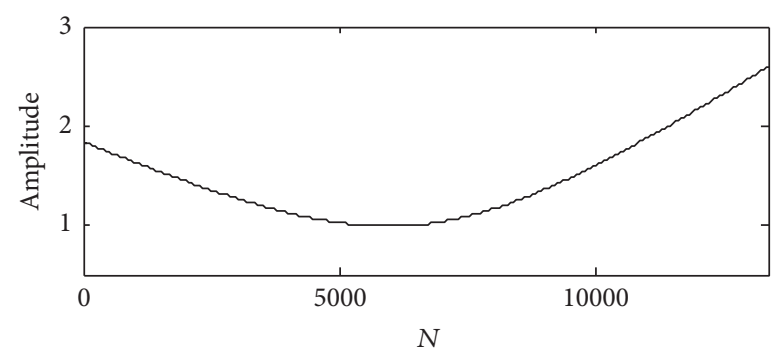

(c)

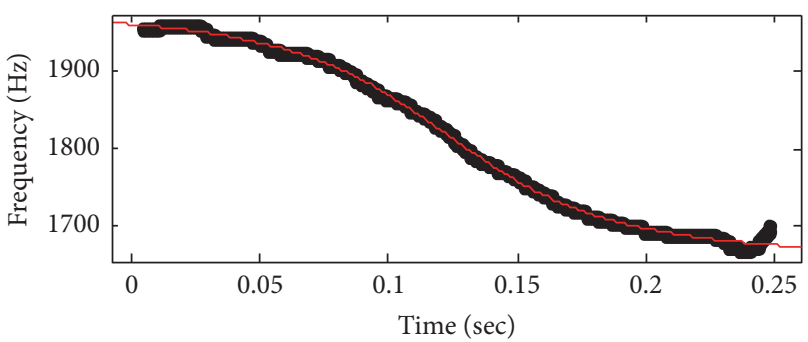

- $\bullet$ - Ridge line

- IF

(b)

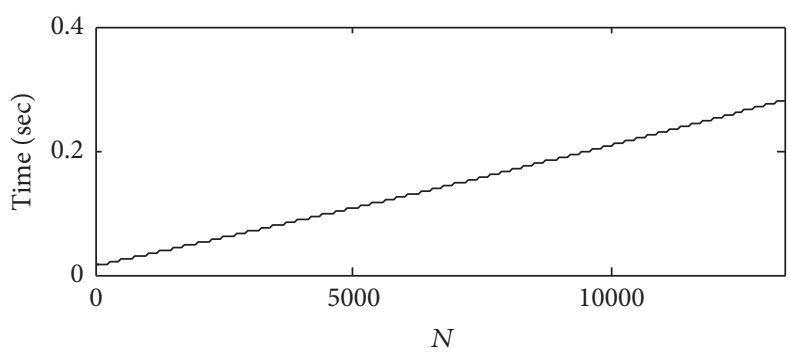

(d)

FIGURE 12: (a) Curve fitting by the method proposed in Section 3. (b) Curve fitting based on STFT. (c) Amplitude demodulation function. (d) Receiving-time vector. 


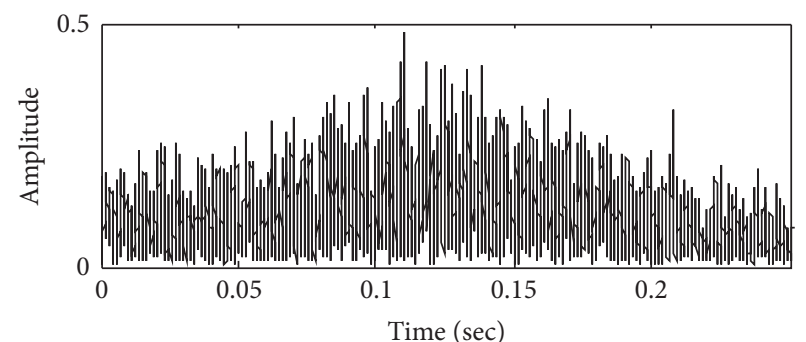

(a)

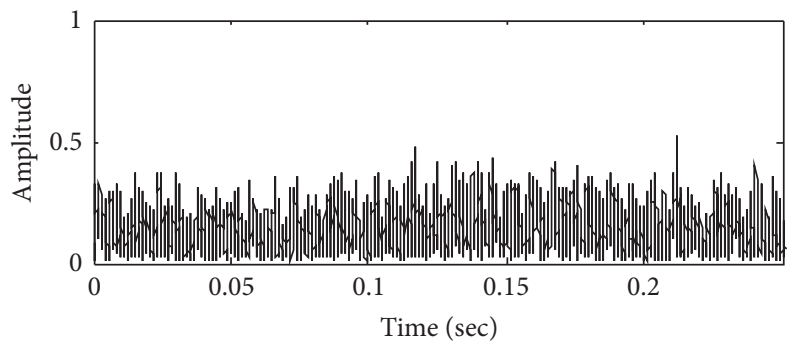

(c)

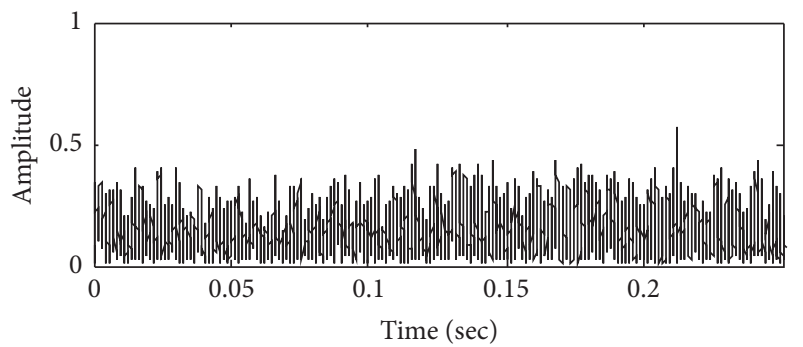

(e)

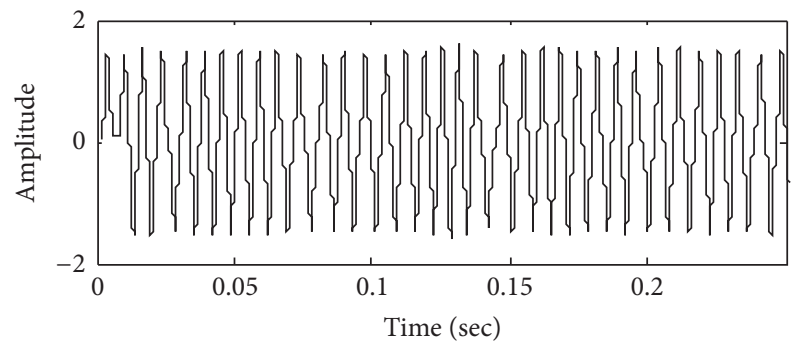

(g)

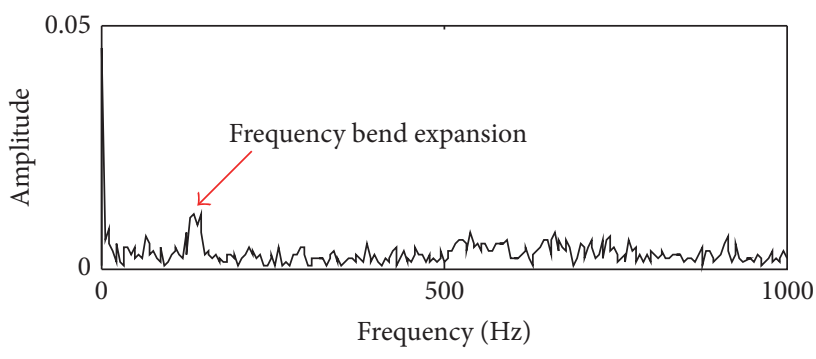

(b)

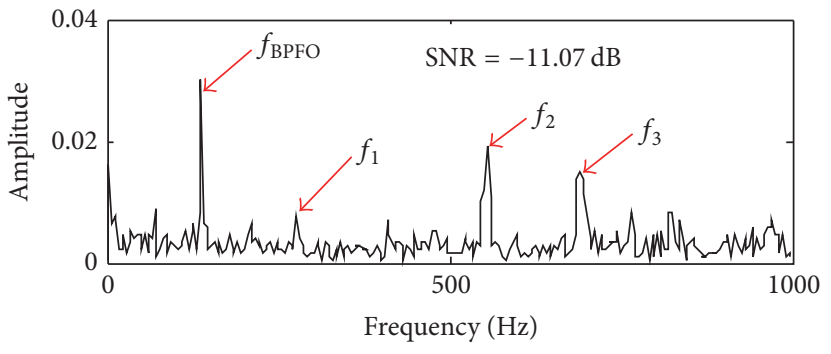

(d)

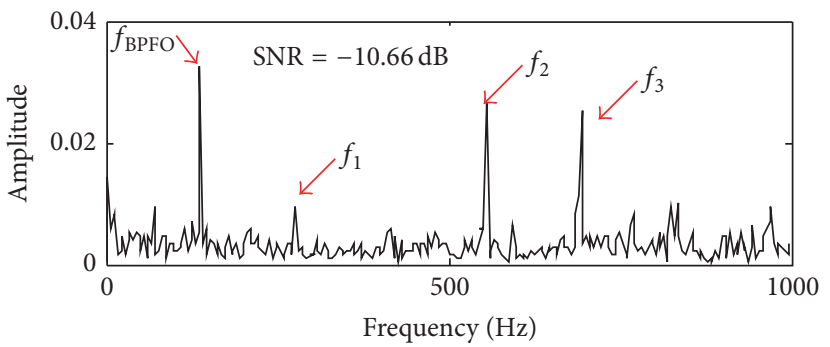

(f)

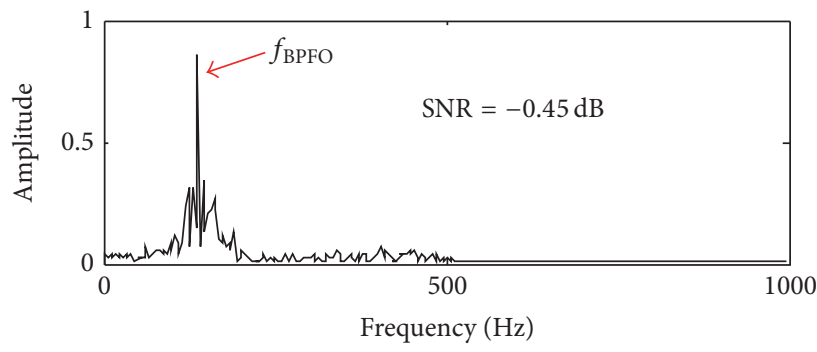

(h)

FIGURE 13: Result of the outer-race-fault signal analysis: (a) and (b) Envelope signal and spectrum of the wayside recorded signal; (c) and (d) the Doppler-free signal based on traditional STFT and envelope spectrum of signal; (e) and (f) the Doppler-free signal based on the improved TIR and envelope spectrum of signal; (g) and (h) WCCSR output signal and spectrum.

The improved TIR method is then employed to analyze this signal. The signal was firstly filtered by a Butterworth band-pass filter with the frequency band of $[1680 \mathrm{~Hz}$, $1850 \mathrm{~Hz}]$ and then handled with STFT to attain a TFD. The TFD of the band-pass signal is shown in Figure 15(a). A major TF curve as well as other frequency components can be seen in the TFD. And also the background noise is heavy. The method explained in Section 3 is then conducted and the results are shown in Figures 15(b)-15(d). Figure 15(b) shows the background noise eliminated by setting an energy threshold of $H=0.0773(k=15, \bar{G}(\omega, \tau)=0.0052)$. The interference elements are eliminated if the length is smaller than $l=18$. The operation process of eliminating interference element is indicated in Figures 15(b) and 15(c). Labels 2 and 4 still remained because their length is large than the set length threshold value. They can be removed by limit of the degree of jump between the searched two adjacent local maximum values during the IF calculation. The obtained IF is shown in Figure 15(d). The parameters could be attained by curve fitting on the IF using (1).

Figure 16 shows the compared result of the proposed method (method (a)) and the STFT method (method (b)). It can also be seen that the TF ridge line extracted using STFT directly fluctuates strongly. Table 3 shows the curve fitting results using the two methods. Results show that the proposed method also achieved a higher accuracy in this 

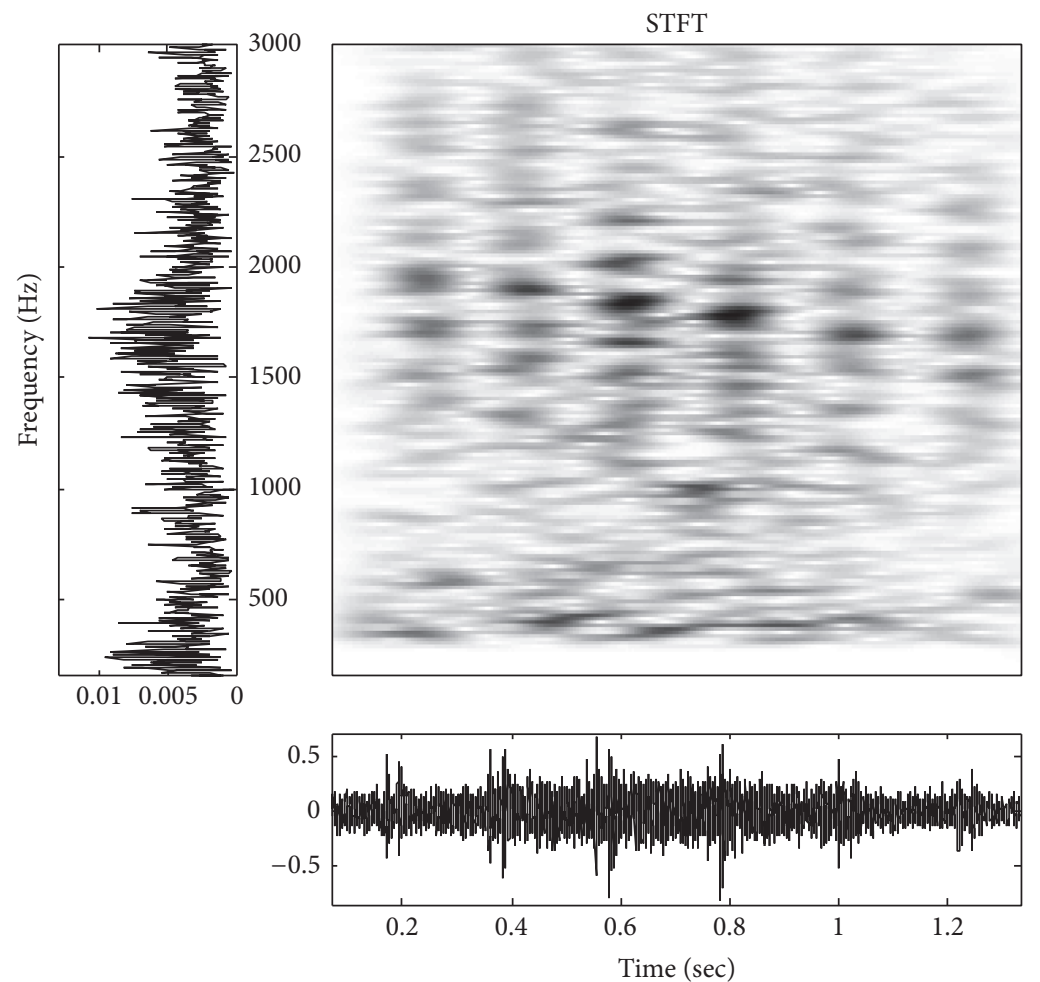

(a)
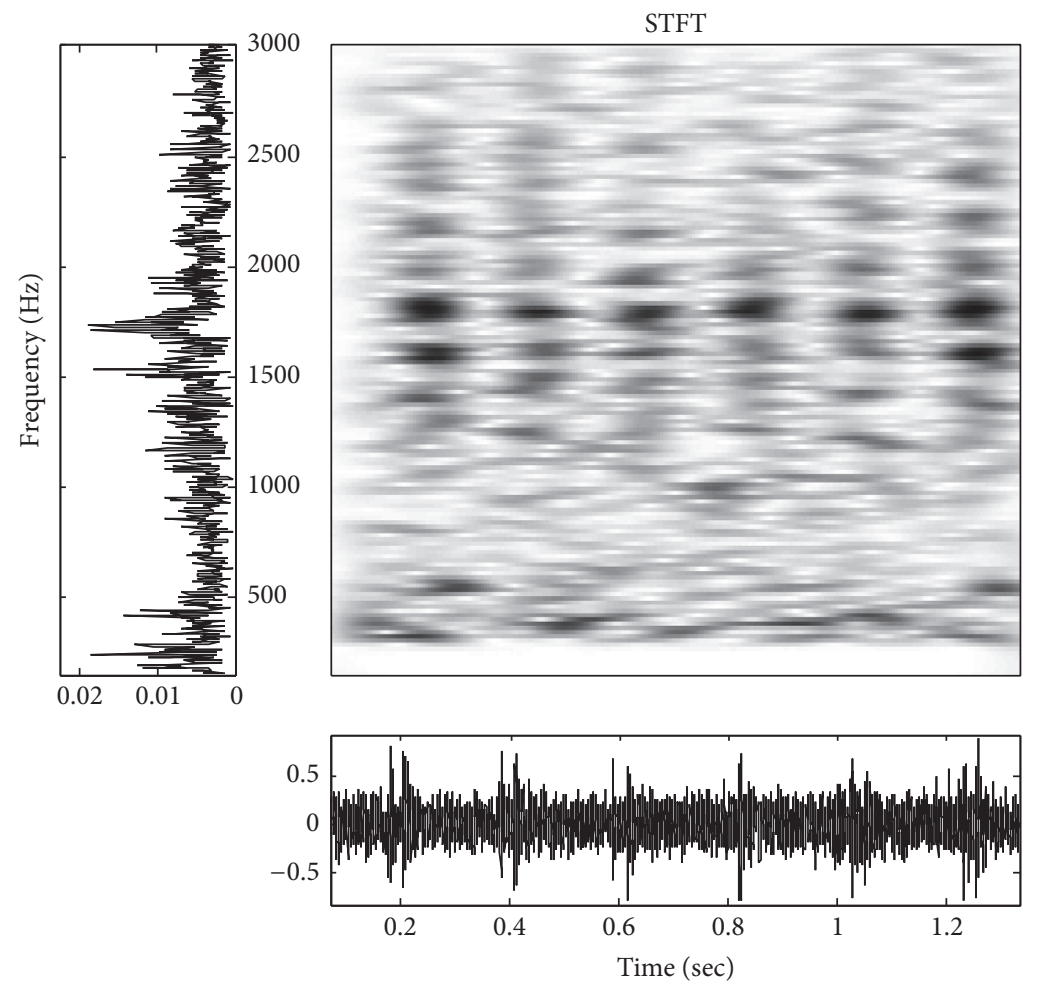

(b)

FIGURE 14: (a) Original signal. (b) Doppler-free signal. 


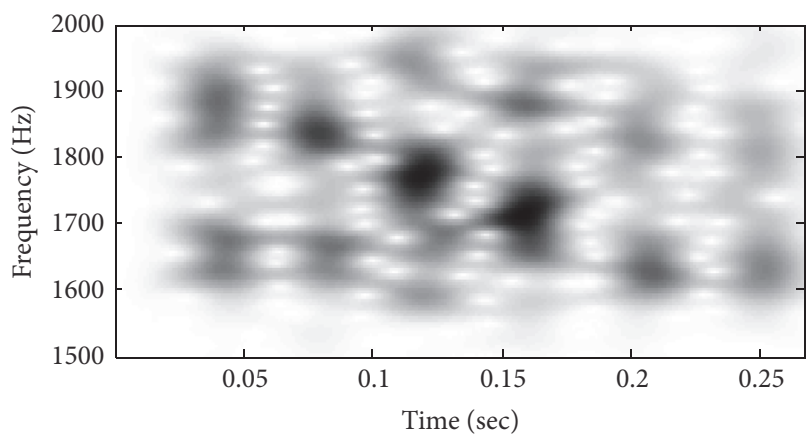

(a)

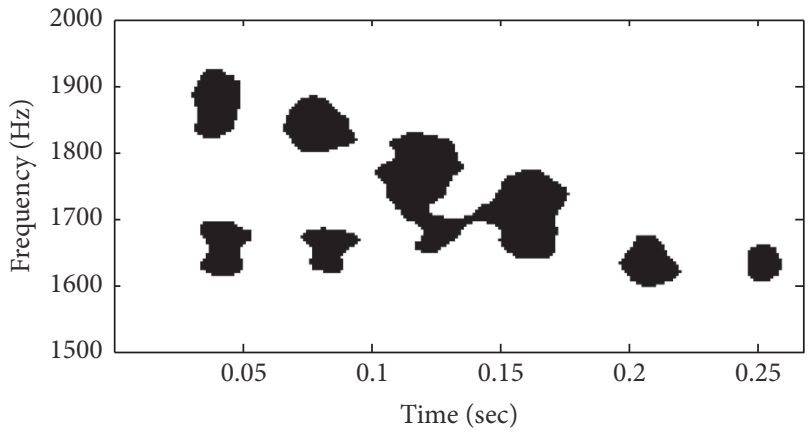

(c)

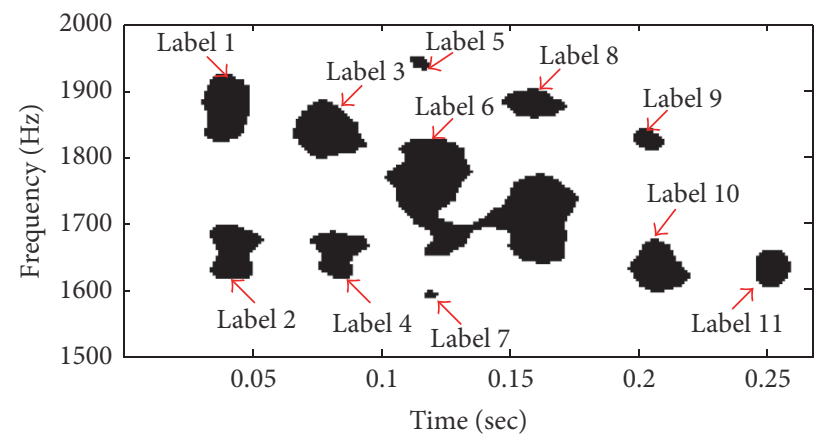

(b)

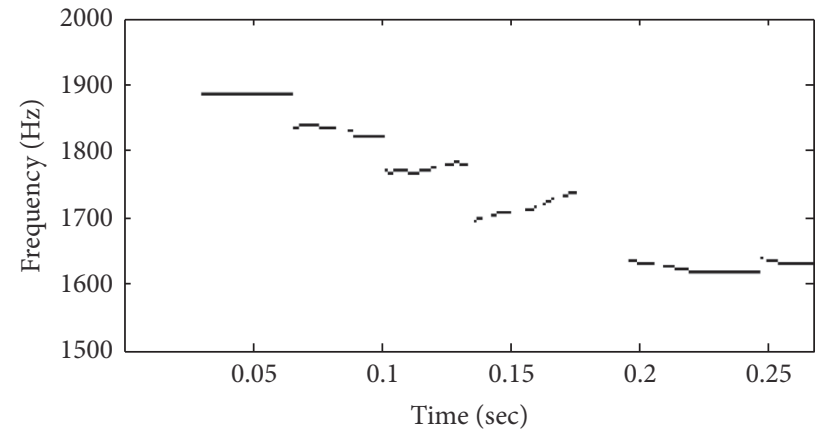

(d)

FIGURE 15: Process of ridge line extraction: (a) band-pass signal; (b) background noise cancellation; (c) interference element cancellation; (d) TF ridge line extraction.

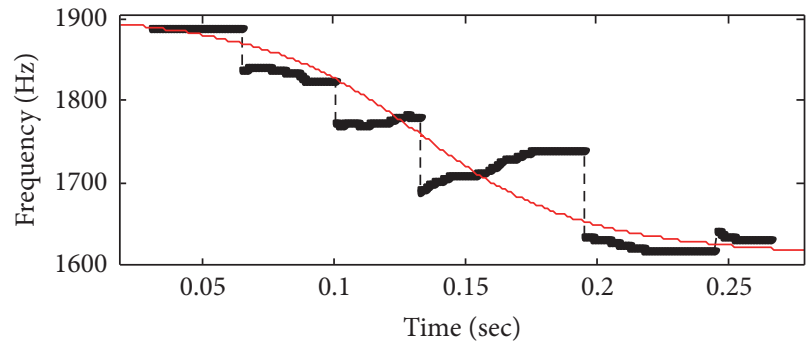

- - Ridge line

- IF

(a)

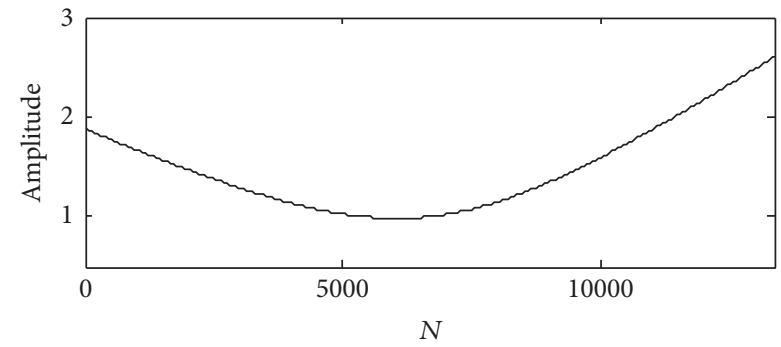

(c)

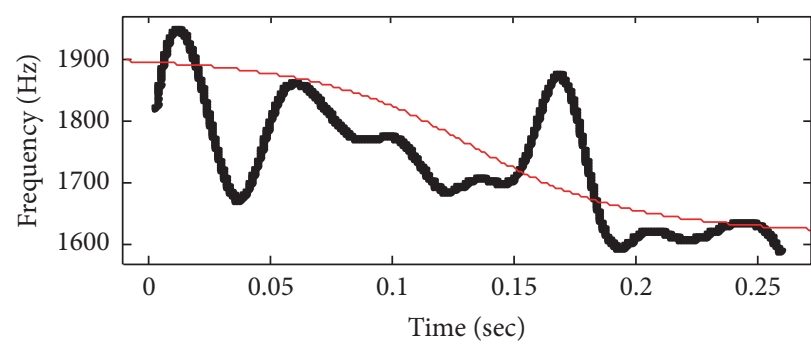

- - Ridge line

— IF

(b)

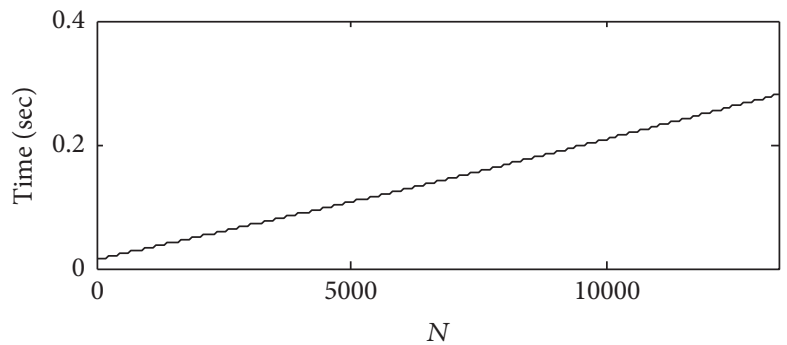

(d)

FIGURE 16: (a) Curve fitting by the method proposed in Section 3. (b) Curve fitting based on STFT. (c) Amplitude demodulation function. (d) Receiving-time vector. 


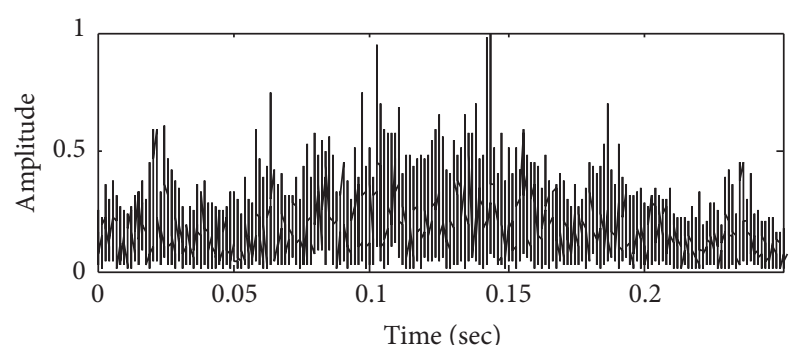

(a)

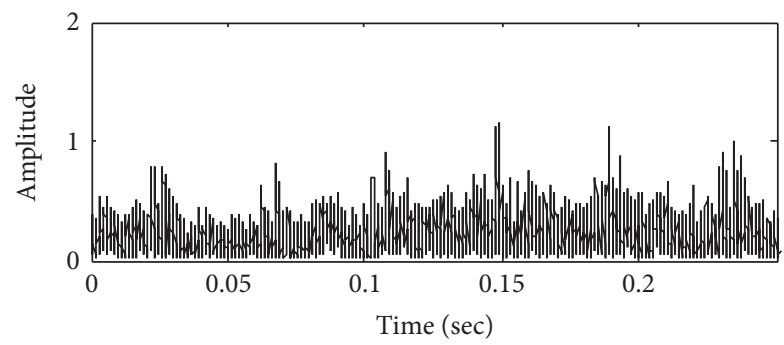

(c)

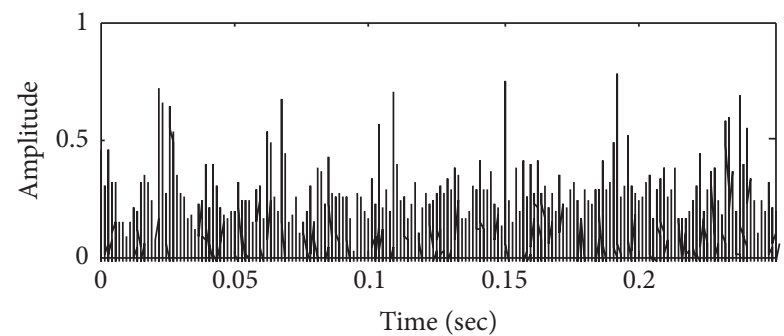

(e)

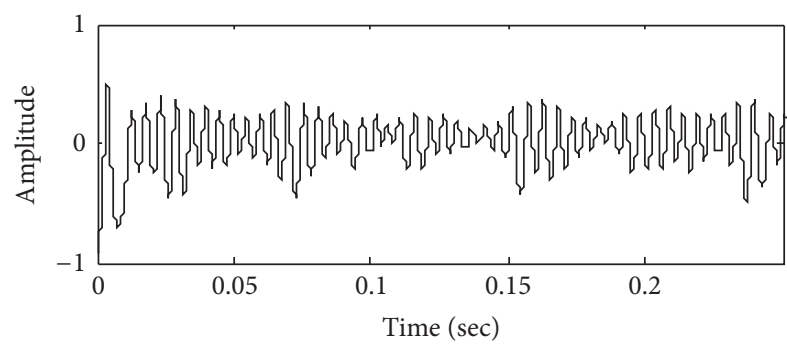

(g)

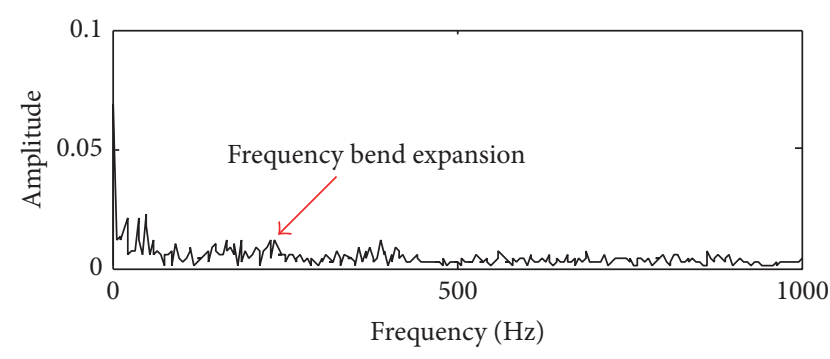

(b)

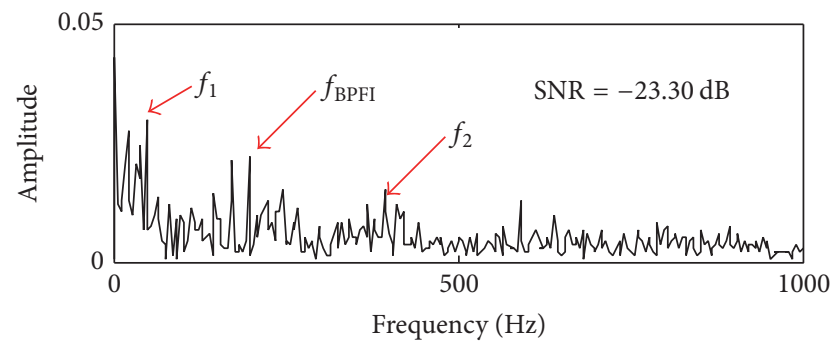

(d)

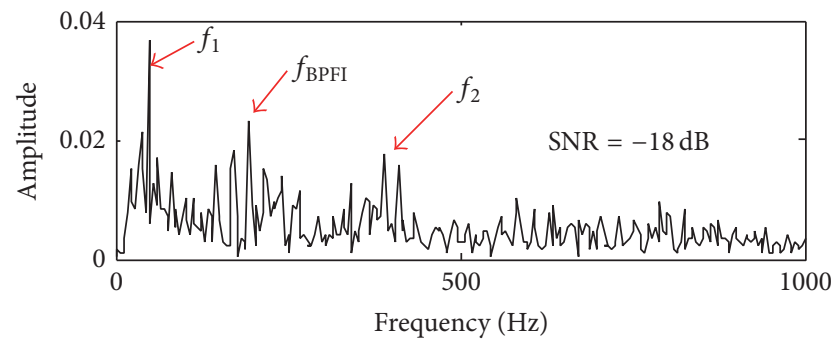

(f)

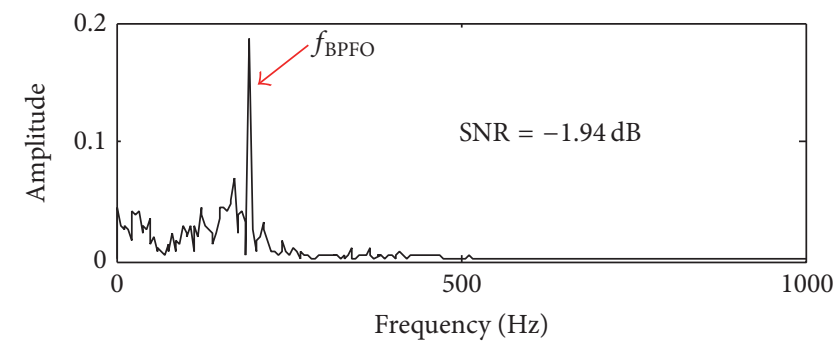

(h)

FIGURE 17: Result of the outer-race-fault signal analysis: (a) and (b) envelope signal and spectrum of the wayside recorded signal; (c) and (d) the Doppler-free signal based on traditional STFT and envelope spectrum of signal; (e) and (f) the Doppler-free signal based on the improved TIR and envelope spectrum of signal; $(\mathrm{g})$ and $(\mathrm{h})$ WCCSR output signal and spectrum.

TABLE 3: Results of parameters estimation by the two methods.

\begin{tabular}{lcccc}
\hline Parameters & $f_{0}$ & $v$ & $s$ & $r$ \\
\hline Actual value & $1800 \mathrm{~Hz}$ & $30 \mathrm{~m} / \mathrm{s}$ & $4 \mathrm{~m}$ & $2 \mathrm{~m}$ \\
Method (a) & $1793 \mathrm{HZ}$ & $30.01 \mathrm{~m} / \mathrm{s}$ & $3.98 \mathrm{~m}$ & $2.01 \mathrm{~m}$ \\
Error (\%) & 0.61 & 0.03 & 0.5 & 0.5 \\
Method (b) & 1760 & 32.12 & 3.85 & 2.21 \\
Error (\%) & 2.22 & 7.06 & 3.75 & 10.5 \\
\hline
\end{tabular}

case too. The estimated parameters are then applied to TIR to reduce the Doppler effect. The amplitude demodulation function and receiving-time vector are shown in Figures 16(c) and 16(d). The obtained Doppler-free signal and its TFD are shown in Figure 14(b). The envelope signal of the obtained Doppler-free signal and its envelope spectrum are shown in Figures 17(e) and 17(f). Comparing with the envelope spectrum of the original signal shown in Figure 17(b), the FCF of the Doppler-free signal is sharp, narrow, and accurate. What is more, the modulation sidebands around the harmonics of the basic fault frequency, spaced at shaft speed for inner-race faults, can be clearly seen which indicates that the phenomenon of FCF expansion has been eliminated. As a comparison, the parameters estimated by the traditional STFT method are also used to reduce the Doppler effect. The 
envelope signal of the obtained Doppler-free signal and its envelope spectrum are shown in Figures 17(c) and 17(d). It can be seen that the rotating frequency and $f_{\mathrm{BPFO}}$ are hardly to be identified which indicates that the Doppler effect has not been eliminated effectively.

However, in practice, the FCF can hardly be identified by unskilled workers because of disturbance of the rotating frequency and the modulation sidebands. The calculated SNR of the FCF is $-18.00 \mathrm{~dB}$. The WCCSR is then employed to amplify the FCF. The optimal output is obtained under the parameters of $h_{\mathrm{opt}}=0.018$ and $\gamma_{\mathrm{opt}}=0.09$. The output waveform and its FFT spectrum are shown in Figures 17(e) and $17(\mathrm{f})$, respectively. It can be seen that $f_{\mathrm{BPFO}}$ is highlighted and can be clearly identified after the WCCSR-based information enhancement. The SNR has been improved to be $-1.94 \mathrm{~dB}$. The results demonstrate that the diagnosisrelevant information is enhanced and the effectiveness and the diagnosis accuracy of the envelope spectrum analysis are thus improved. The analysis results in this section have further verified the effectiveness of the proposed parameters estimation method as well as the information enhancement method.

\section{Conclusion}

This study proposes solutions for the two problems, namely, the Doppler effect reduction and the weak information enhancement, in the envelope spectrum analysis of the wayside bearing acoustic signal. The proposed method is based on the theoretical background of TIR and WCCSR. As a pavement of the TIR, a new TF ridge line extraction method based on the maximum searching algorithm is proposed to estimate the kinematic parameters. A SRbased signal amplification method is introduced to enhance the weak information. The proposed WCC criterion can guide the output both in time and frequency domain so that the amplification can be conducted adaptively. The advantage of the proposed improved TIR method is the kinematic parameters estimation prior to the time-domain interpolation process. The improvement of conditional TIR overcomes the limitation of the traditional TIR, in which the receiving-time vector is calculated based on the kinematic parameters that should be known beforehand. Thus, the agility and convenience of TIR are improved. A simulated signal is processed and the ridge line is obtained to verify the effectiveness of the new TF ridge line extraction and the parameters estimation using curve fitting. On the other hand, the proposed WCCSR method can highlight the FCF after the Doppler effect has been removed. This process can enhance the weak diagnosis information as much as possible. Experimental cases using a real train wheel bearing are also carried out to further verify the effectiveness of the proposed method. The proposed method may be applicable to practical wayside acoustic bearing detection system to enhance the diagnosis performance. However, the proposed method still has deviation, especially when dealing with inner-race signal because of the discontinuous shock and insufficient quantity of inner-race signal. The accuracy of TF ridge line extraction will be enhanced in the next exploration. And, in practice, the noise must be heavier than the cases studied in this paper, so a real wayside recorded signal from a real train is necessary to be analyzed to further confirm the effectiveness of the WCCSR method.

\section{Competing Interests}

The authors declare that there is no conflict of interests regarding the publication of this paper.

\section{Acknowledgments}

This work is supported by the National Natural Science Foundation of China (no. 51505001, no. 51675001, and no. 51605002) and the Natural Science Foundation of Anhui Province (no. 1508085SQE212 and no. 1608085QE110).

\section{References}

[1] J. E. Cline, J. R. Bilodeau, and R. L. Smith, "Acoustic wayside identification of freight car roller bearing defects," in Proceedings of the ASME/IEEE Joint Railroad Conference, pp. 79-83, Philadelphia, Pa, USA, 1998.

[2] C. Southern, D. Rennison, and U. Kopke, "RailBAM-An advanced bearing acoustic monitor: initial operational performance results," in Proceedings of Conference on Railway Engineering, pp. 23.01-23.07, Darwin, Australia, June 2004.

[3] O. D. Snell and I. Nairne, "Acoustic bearing monitoring-the future RCM 2008," in Proceedings of the 4th IET International Conference on Railway Condition Monitoring (RCM '08), June 2008.

[4] H. C. Choe, Y. Wan, and A. K. Chan, "Neural pattern identification of railroad wheel-bearing faults from audible acoustic signals: comparison of FFT, CWT, and DWT features," in Proceedings of the Image Compression and Processing, vol. 3078 of Proceedings of SPIE, Orlando, Fla, USA, April 1997.

[5] J. Dybała and S. Radkowski, "Reduction of Doppler effect for the needs of wayside condition monitoring system of railway vehicles," Mechanical Systems and Signal Processing, vol. 38, no. 1, pp. 125-136, 2013.

[6] P. Borghesani, R. Ricci, S. Chatterton, and P. Pennacchi, "A new procedure for using envelope analysis for rolling element bearing diagnostics in variable operating conditions," Mechanical Systems and Signal Processing, vol. 38, no. 1, pp. 23-35, 2013.

[7] R. B. Randall and J. Antoni, "Rolling element bearing diagnostics-a tutorial," Mechanical Systems and Signal Processing, vol. 25, no. 2, pp. 485-520, 2011.

[8] F. Liu, Q. He, F. Kong, and Y. Liu, "Doppler effect reduction based on time-domain interpolation resampling for wayside acoustic defective bearing detector system," Mechanical Systems and Signal Processing, vol. 46, no. 2, pp. 253-271, 2014.

[9] A. Zhang, F. Hu, Q. He, C. Shen, F. Liu, and F. Kong, "Doppler shift removal based on instantaneous frequency estimation for wayside fault diagnosis of train bearings," Journal of Vibration and Acoustics, vol. 136, no. 2, Article ID 021019, 2014.

[10] I. Pitas, Nonlinear Digital Filters: Principles and Applications, Kluwer International, 1990.

[11] P. P. Vaidyanathan, "Multirate digital filters, filter banks, polyphase networks, and applications: a tutorial," Proceedings of the IEEE, vol. 78, no. 1, pp. 56-93, 1990. 
[12] P. W. Tse and D. Wang, "The automatic selection of an optimal wavelet filter and its enhancement by the new sparsogram for bearing fault detection: part 2 of the two related manuscripts that have a joint title as 'two automatic vibration-based fault diagnostic methods using the novel sparsity measurementparts 1 and 2,' Mechanical Systems \& Signal Processing, vol. 40, no. 2, pp. 520-544, 2013.

[13] Z. K. Peng and F. L. Chu, "Application of the wavelet transform in machine condition monitoring and fault diagnostics: a review with bibliography," Mechanical Systems \& Signal Processing, vol. 18, no. 2, pp. 199-221, 2004.

[14] R. Yan, R. X. Gao, and X. Chen, "Wavelets for fault diagnosis of rotary machines: a review with applications," Signal Processing, vol. 96, pp. 1-15, 2014.

[15] S. Lu, Q. He, F. Hu, and F. Kong, "Sequential multiscale noise tuning stochastic resonance for train bearing fault diagnosis in an embedded system," IEEE Transactions on Instrumentation and Measurement, vol. 63, no. 1, pp. 106-116, 2014.

[16] L. Gammaitoni, P. Hänggi, P. Jung, and F. Marchesoni, "Stochastic resonance," Reviews of Modern Physics, vol. 70, no. 1, pp. 223287, 1998.

[17] Z. Zhang, J. Wang, and H. Zha, "Adaptive manifold learning," IEEE Transactions on Pattern Analysis and Machine Intelligence, vol. 34, no. 2, pp. 253-265, 2012.

[18] J. Yu, "Local and nonlocal preserving projection for bearing defect classification and performance assessment," IEEE Transactions on Industrial Electronics, vol. 59, no. 5, pp. 2363-2376, 2012.

[19] Y. Dong, M. Liao, X. Zhang, and F. Wang, "Faults diagnosis of rolling element bearings based on modified morphological method," Mechanical Systems and Signal Processing, vol. 25, no. 4, pp. 1276-1286, 2011.

[20] D. Wang, Y. L. Tse, and P. W. Tse, "A morphogram with the optimal selection of parameters used in morphological analysis for enhancing the ability in bearing fault diagnosis," Measurement Science \& Technology, vol. 23, no. 6, pp. 65001-65015, 2012.

[21] J. Wang, Q. He, and F. Kong, "Adaptive multiscale noise tuning stochastic resonance for health diagnosis of rolling element bearings," IEEE Transactions on Instrumentation and Measurement, vol. 64, no. 2, pp. 564-577, 2015.

[22] P. M. Morse and K. U. Ingard, Theoretical Acoustics, Princeton University Press, Princeton, NJ, USA, 1986.

[23] B. F. Hlawatsch and G. F. Boudreaux-Bartels, "Linear and quadratic timefrequency representations," IEEE Signal Processing Magazine, vol. 9, no. 2, pp. 21-67, 2010.

[24] R. X. Gao and R. Yan, Wavelets: Theory and Applications for Manufacturing, Springer Science \& Business Media, 2010.

[25] L. Durak and O. Arikan, "Short-time Fourier transform: two fundamental properties and an optimal implementation," IEEE Transactions on Signal Processing, vol. 51, no. 5, pp. 1231-1242, 2003.

[26] S. H. Nawab and T. F. Quatieri, "Short-time Fourier transform," in Advanced Topics in Signal Processing, pp. 289-337, 1987.

[27] L. Cohen, "Time-frequency distributions-a review," Proceedings of the IEEE, vol. 77, no. 7, pp. 941-981, 1989.

[28] S. Xue, H. Cheng, and Y. Yang, "Application of pseudo WignerVille distribution to fault diagnosis in motor bearing," Mechanical Engineering \& Automation, vol. 4, pp. 98-100, 2008.

[29] R. Yan and R. X. Gao, "Hilbert-huang transform-based vibration signal analysis for machine health monitoring," IEEE Transactions on Instrumentation and Measurement, vol. 55, no. 6, pp. 2320-2329, 2006.
[30] R. Yan and R. X. Gao, "Transient signal analysis based on Hilbert-Huang transform," in Proceedings of the IEEE Instrumentation and Measurement Technology Conference (IMTC '05), pp. 1198-1202, May 2005.

[31] R. P. Grzeszczuk and D. N. Levin, "Brownian strings': segmenting images with stochastically deformable contours," IEEE Transactions on Pattern Analysis and Machine Intelligence, vol. 19, no. 10, pp. 1100-1114, 1997.

[32] R. L. Stevenson, "Region-based color image segmentation scheme," in Proceedings of the International Society for Optical Engineering, vol. 3653 of Proceedings of SPIE, pp. 537-540, San Jose, Calif, USA, January 1999.

[33] S. Lu, Q. He, H. Zhang, and F. Kong, "Enhanced rotating machine fault diagnosis based on time-delayed feedback stochastic resonance," Journal of Vibration and Acoustics, vol. 137, no. 5, Article ID 051008, 2015.

[34] S. Lu, Q. He, D. Dai, and F. Kong, "Periodic fault signal enhancement in rotating machine vibrations via stochastic resonance," Journal of Vibration and Control, vol. 22, no. 20, pp. 4227-4246, 2016. 


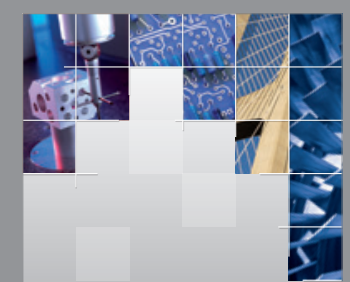

\section{Enfincering}
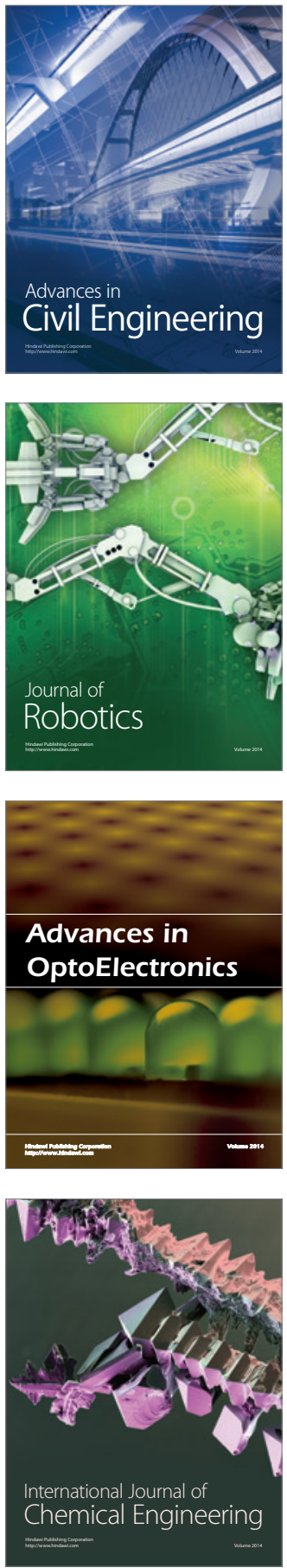

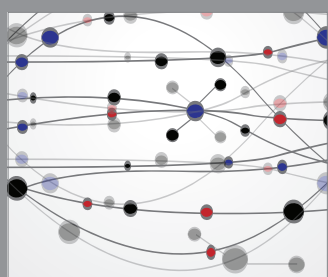

The Scientific World Journal

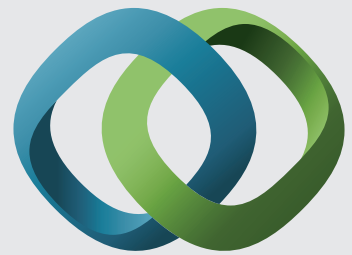

\section{Hindawi}

Submit your manuscripts at

https://www.hindawi.com
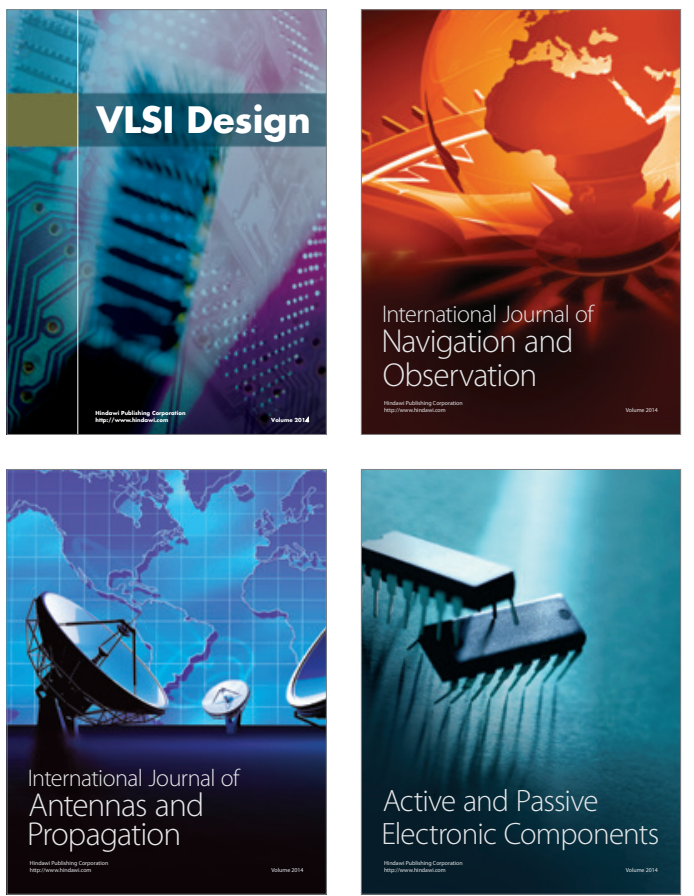
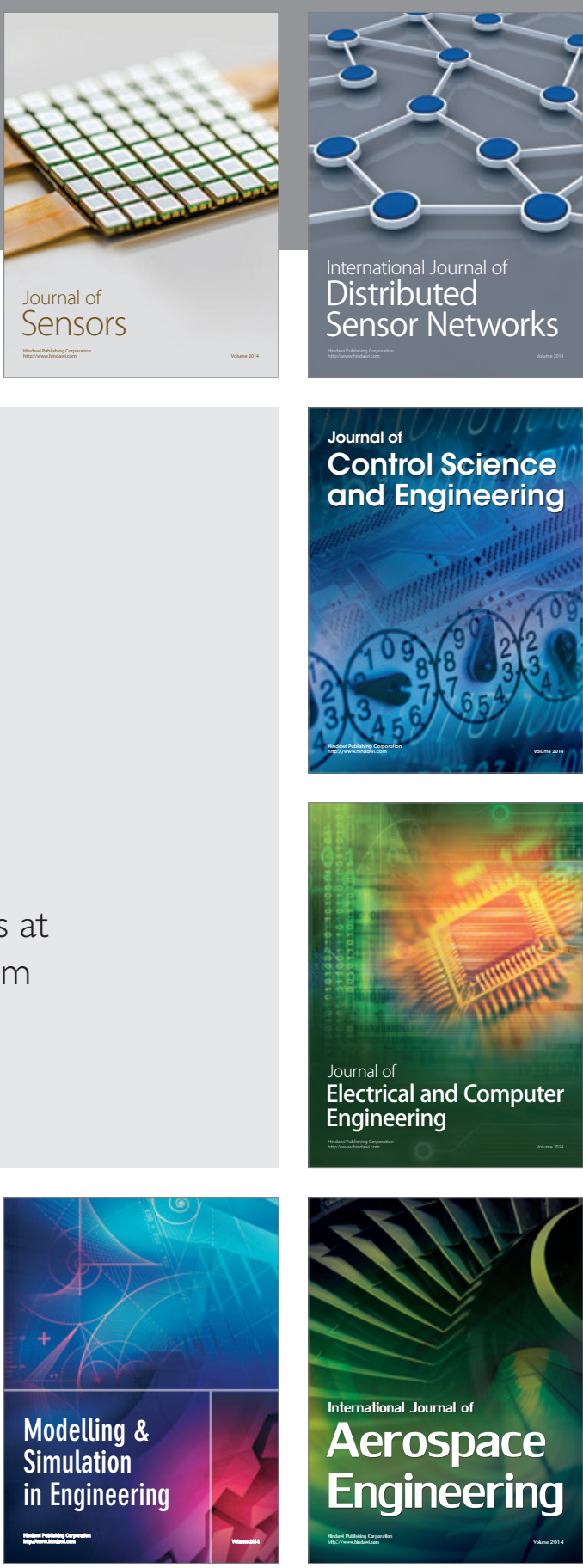

International Journal of

Distributed

Sensor Networks

$-$

Joumal of

Control Science

and Engineering
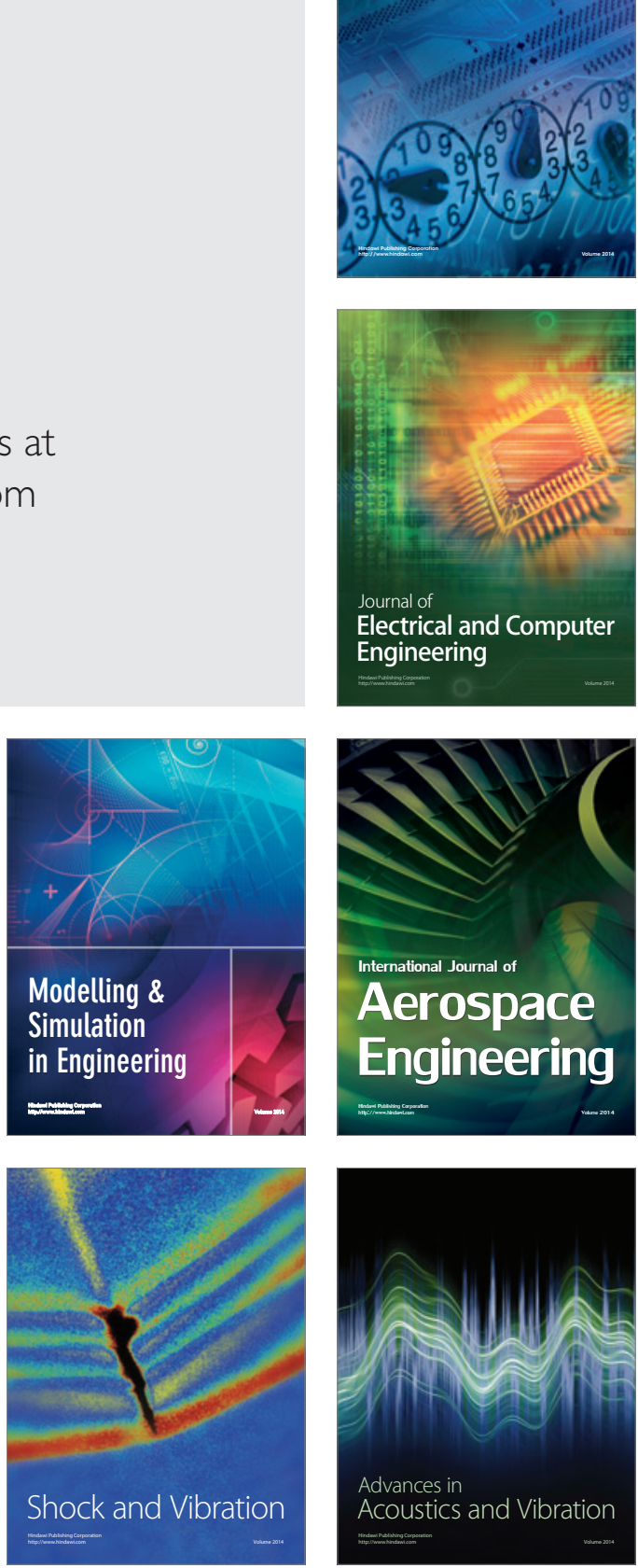